\title{
Non-tachyonic semi-realistic non-supersymmetric heterotic-string vacua
}

\author{
Johar M. Ashfaque ${ }^{\mathrm{a}}$, Panos Athanasopoulos ${ }^{\mathrm{b}}$, Alon E. Faraggi ${ }^{\mathrm{c}}$, Hasan Sonmez ${ }^{\mathrm{d}}$ \\ Department of Mathematical Sciences, University of Liverpool, Liverpool L69 7ZL, UK
}

Received: 1 December 2015 / Accepted: 31 March 2016 / Published online: 15 April 2016

(c) The Author(s) 2016. This article is published with open access at Springerlink.com

\begin{abstract}
The heterotic-string models in the free fermionic formulation gave rise to some of the most realistic-string models to date, which possess $N=1$ spacetime supersymmetry. Lack of evidence for supersymmetry at the LHC instigated recent interest in non-supersymmetric heterotic-string vacua. We explore what may be learned in this context from the quasi-realistic free fermionic models. We show that constructions with a low number of families give rise to proliferation of a priori tachyon producing sectors, compared to the non-realistic examples, which typically may contain only one such sector. The reason being that in the realistic cases the internal six dimensional space is fragmented into smaller units. We present one example of a quasi-realistic, nonsupersymmetric, non-tachyonic, heterotic-string vacuum and compare the structure of its massless spectrum to the corresponding supersymmetric vacuum. While in some sectors supersymmetry is broken explicitly, i.e. the bosonic and fermionic sectors produce massless and massive states, other sectors, and in particular those leading to the chiral families, continue to exhibit Fermi-Bose degeneracy. In these sectors the massless spectrum, as compared to the supersymmetric cases, will only differ in some local or global $U(1)$ charges. We discuss the conditions for obtaining $n_{b}=n_{f}$ at the massless level in these models. Our example model contains an anomalous $U(1)$ symmetry, which generates a tadpole diagram at one-loop order in string perturbation theory. We speculate that this tadpole diagram may cancel the corresponding diagram generated by the one-loop non-vanishing vacuum energy and that in this respect the supersymmetric and non-supersymmetric vacua should be regarded on an equal footing. Finally we discuss vacua that contain two supersymmetry generating sectors.
\end{abstract}

\footnotetext{
a e-mail: jauhar@liv.ac.uk

be-mail:panos@liv.ac.uk

c e-mail: alon.faraggi@liv.ac.uk

d e-mail: Hasan.Sonmez@liv.ac.uk
}

\section{Introduction}

The discovery of the agent of electroweak symmetry breaking at the LHC $[1,2]$ is a pivotal moment in particle physics. While confirmation of this agent as the Standard Model electroweak doublet representation will require experimental scrutiny in the decades to come, the data to date seems to vindicate this possibility. Substantiation of this interpretation of the data will reinforce the view that the electroweak symmetry breaking mechanism is intrinsically perturbative, and that the SM provides a viable perturbative parameterisation up to the Planck scale. Moreover, the large scale unification scenario is further motivated by the embedding of the SM matter states in the chiral $S O(10)$ representation; by the logarithmic evolution of the SM parameters; by proton longevity; and by the suppression of left-handed neutrino masses. Gaining further insight into the fundamental origins of the SM parameters can then only be obtained by incorporating gravity into the picture.

String theory provides the most developed contemporary approach to study how the Standard Model parameters may arise from a unified theory of the gauge and gravitational interactions. For this purpose several models that reproduce the spectrum of the Minimal Supersymmetric Standard Model have been produced [3-8]. Amongst them the free fermionic models [3-5,9-23] are the most studied examples. The heterotic string in particular provides a compelling framework to study the gauge-gravity synthesis in the large scale unification scenario, as it reproduces the embedding of the SM chiral spectrum in spinorial $S O(10)$ representations.

The majority of semi-realistic heterotic-string models constructed to date possess $N=1$ spacetime supersymmetry, while non-supersymmetric vacua were investigated sporadically [24-31]. In the absence of evidence of supersymmetry at the LHC recent interest in non-supersymmetric heterotic-string vacua has emerged [32-38]. It is therefore prudent to examine what may be learned in that context from the quasi-realistic free fermionic models. In this paper this 
question is considered. We discuss the different avenues that may be used to break supersymmetry directly at the string scale and how they compare with the recent analysis [34].

Our paper is organised as follows: in Sect. 2, we review the structure of the phenomenological free fermionic heteroticstring models. In Sect. 3, we discuss the phases that break supersymmetry in the string models and the different patterns that they induce. Further discussion of the existence of sectors producing tachyons in these models and the relation of the abundance of these sectors with the number of families is given. Moreover, in Sect. 4 a non-supersymmetric tachyonfree model is presented and its relation to the supersymmetric counterpart is inferred. In Sect. 5 we discuss the construction of string vacua with split supersymmetry, in which supersymmetry is produced by two sectors. Section 6 contains our conclusions.

\section{Phenomenological free fermionic models}

In this section, we review the structure of the phenomenological free fermionic models. It should be stressed that these free fermionic models correspond to $Z_{2} \times Z_{2}$ toroidal orbifolds and their phenomenological characteristics are deeply rooted in the structure of the $Z_{2} \times Z_{2}$ orbifolds. In this respect, the free fermionic formalism merely provides an accessible set of tools to extract the spectra of the string vacua and their properties. Furthermore, the free fermionic machinery extends to the massive string spectrum via the analysis of the relevant partition function. This provides important insight into the symmetries that underly the string landscape and eventually may prove instrumental in understanding how the string vacuum is selected. However, one should not tie the cart before the horse. The fermionic and bosonic representations only provide complementary tools that are formally identical in two dimensions. The physically relevant properties of these free fermionic models are due to their underlying $Z_{2} \times Z_{2}$ orbifold structure.

In the free fermionic formulation of the heterotic string in four dimensions, all the extra degrees of freedom needed to cancel the conformal anomaly are represented as free fermions propagating on the string worldsheet. It is important to note that the two dimensional fermions are free only at a special point in the moduli space [39]. However, the models can be deformed away from that point by incorporating the moduli as worldsheet Thirring interactions [40-42]. Since the twisted matter spectrum of the $Z_{2} \times Z_{2}$ orbifolds, which gives rise to the Standard Model matter states, is independent of the moduli, working at the free fermionic point is just a convenient choice. In the light-cone gauge the supersymmetric left-moving sector includes the two transverse spacetime fermionic coordinates $\psi^{\mu}$ and 18 internal worldsheet real fermions $\chi^{I}$, whereas the right-moving bosonic sector con- tains 44 real worldsheet fermions $\phi^{a}$. The worldsheet supersymmetry is realised non-linearly in the left-moving sector and the worldsheet supercurrent is given by

$T_{F}=\psi^{\mu} \partial X_{\mu}+f_{I J K} \chi^{I} \chi^{J} \chi^{K}$,

where $f_{I J K}$ are the structure constants of the 18 dimensional semi-simple Lie group. The 18 left-moving worldsheet fermions $\chi^{I}$ transform in the adjoint representation of the Lie group, which in the case of the fermionic $Z_{2} \times Z_{2}$ orbifolds with $N=1$ SUSY is $S U(2)^{6}$. Such models provide our starting point and we will discuss in later sections how supersymmetry is broken. The $\chi^{I}$ therefore transform in the adjoint representation of $S U(2)^{6}$, and they are denoted by $\chi^{I}, y^{I}, \omega^{I}$ with $I=1, \ldots, 6$. Under parallel transport around a non-contractible loop of the one-loop vacuum to vacuum amplitude the worldsheet fermions pick up a phase

$f \rightarrow-\mathrm{e}^{i \pi \alpha(f)} f$,

with $\alpha(f) \in(-1,+1]$. The phases for all worldsheet fermions constitute the spin structure of the models and are given in the form of 64 dimensional boundary condition basis vectors. The partition function,

$Z(\tau)=\sum_{\alpha, \beta \in \Xi} c\left(\begin{array}{l}\alpha \\ \beta\end{array}\right) \operatorname{Tr}\left(\begin{array}{l}\alpha \\ \beta\end{array}\right)$,

is a sum over all spin structures, where $c\left(\begin{array}{l}\alpha \\ \beta\end{array}\right)$ are Generalised GSO (GGSO) projection coefficients and $\operatorname{Tr}\left(\begin{array}{l}\alpha \\ \beta\end{array}\right) \equiv$ $\operatorname{Tr}\left(\mathrm{e}^{\mathrm{i} \pi \beta \mathrm{F}_{\alpha}} \mathrm{e}^{\mathrm{i} \pi \tau \mathrm{H}_{\alpha}}\right)$ with $H_{\alpha}$ being the hamiltonian, is the trace over the mode excitations of the worldsheet fields in the sector $\alpha$, subject to the GSO projections induced by the sector $\beta$. Requiring invariance under modular transformations results in a set of constraints on the allowed spin structures and the GGSO projection coefficients. The Hilbert space of a given sector $\alpha$ in the finite abelian additive group $\alpha \in \Xi=\sum_{k} n_{i} b_{i}$, where $n_{i}=0, \ldots, g_{z_{i}}-1$, is obtained by acting on the vacuum of the sector $\alpha$ with bosonic, as well as fermionic oscillators with frequencies $v_{f}, v_{f}^{*}$, and subsequently imposing the GGSO projections

$$
\left\{e^{i \pi\left(b_{i} F_{\alpha}\right)}-\delta_{\alpha} c^{*}\left(\begin{array}{c}
\alpha \\
b_{i}
\end{array}\right)\right\}|s\rangle_{\alpha}=0
$$

with

$\left(b_{i} F_{\alpha}\right) \equiv\left\{\sum_{\substack{\text { real }+ \text { complex } \\ \text { left }}}-\sum_{\substack{\text { real }+ \text { complex } \\ \text { right }}}\right\}\left(b_{i}(f) F_{\alpha}(f)\right)$

where $\delta_{\alpha}$ is the spacetime spin statistics index and $F_{\alpha}(f)$ is a fermion number operator, counting each mode of $f$ once (and if $f$ is complex, $f^{*}$ minus once). For Ramond fermions with $\alpha(f)=1$ the vacuum is a doubly degenerate spinor $| \pm\rangle$, annihilated by the zero modes $f_{0}$ and $f_{0}^{*}$, and with fermion 
numbers $F(f)=0,-1$. The physical states in the string spectrum satisfy the level matching condition

$M_{L}^{2}=-\frac{1}{2}+\frac{\alpha_{L} \cdot \alpha_{L}}{8}+N_{L}=-1+\frac{\alpha_{R} \cdot \alpha_{R}}{8}+N_{R}=M_{R}^{2}$

where $\alpha=\left(\alpha_{L} ; \alpha_{R}\right) \in \Xi$ is a sector in the additive group, and

$N_{L}=\sum_{f}\left(v_{L}\right) ; \quad N_{R}=\sum_{f}\left(v_{R}\right) ;$

$v_{f}=\frac{1+\alpha(f)}{2} ; \quad v_{f^{*}}=\frac{1-\alpha(f)}{2}$.

The $U(1)$ charges with respect to the Cartan generators of the gauge group in four dimensions are given by

$Q(f)=\frac{1}{2} \alpha(f)+F(f)$,

for each complex right-moving fermion $f$. In the usual notation the 64 worldsheet fermions in the light-cone gauge are denoted as $\psi^{\mu}, \chi^{1, \ldots, 6}, y^{1, \ldots, 6}, \omega^{1, \ldots, 6}$ and $\bar{y}^{1, \ldots, 6}, \bar{\omega}^{1, \ldots, 6}$, $\bar{\psi}^{1, \ldots, 5}, \bar{\eta}^{1,2,3}, \bar{\phi}^{1, \ldots, 8}$. Further details on the formalism and notation used in the free fermionic construction can be found in the literature [9-19,43-50].

\subsection{Construction of phenomenological models}

Phenomenological free fermionic heterotic-string models were constructed following two main routes. The first are the so-called NAHE-based models. This set of models utilise a set of eight or nine boundary condition basis vectors. The first five consist of the so-called NAHE set [51] and are common in all these models. The basis vectors underlying the NAHE-based models therefore differ by the additional three or four basis vectors that extend the NAHE set.

The second route follows from the classification methodology that was developed in [52] for the classification of type II free fermionic superstrings and adopted in [18-21,23,4648] for the classification of free fermionic heterotic-string vacua with $S O(10)$ GUT symmetry and its Pati-Salam [20,21] and flipped $S U(5)$ [23] subgroups. The main difference between the two classes of models is that while the NAHE-based models allow for asymmetric boundary conditions with respect to the set of internal fermions $\{y, \omega \mid \bar{y}, \bar{\omega}\}$, the classification method only utilises symmetric boundary conditions. This distinction affects the moduli spaces of the models [53], which can be entirely fixed in the former case [54] but not in the later. On the other hand the classification method enables the systematic scan of spaces of the order of $10^{12}$ vacua, and led to the discovery of spinor-vector duality [46-48,55-59] and exophobic heterotic-string vacua $[20,21]$. In this paper, for reasons that will be clarified below, our discussion is focussed on the NAHE-based models.

\subsubsection{The NAHE set}

The NAHE set [51] is a set of five boundary condition basis vectors $\left\{\mathbf{1}, S, b_{1}, b_{2}, b_{3}\right\}$. With ' 1 ' indicating Ramond boundary conditions and ' 0 ' indicating Neveu-Schwarz boundary conditions. The NAHE-set basis vectors are given by

\begin{tabular}{|c|c|c|c|c|c|}
\hline & $\psi^{\mu} \mid \chi^{12}$ & $\chi^{34} \chi^{56}$ & \begin{tabular}{l|l|}
$\bar{\psi}^{1, \ldots, 5}$ & $\bar{\eta}^{1} \bar{\eta}^{2} \bar{\eta}^{3}$ \\
\end{tabular} & 8 & \\
\hline $\mathbf{1}$ & 1 & 1 & \begin{tabular}{|l|lll|l}
$1, \ldots, 1$ & 1 & 1 & 1 & 1
\end{tabular} & $\overline{1, \ldots, 1}$ & \\
\hline$S$ & 1 & 1 & \begin{tabular}{|l|lll|l}
$0, \ldots, 0$ & 0 & 0 & 0 & 0
\end{tabular} & $0, \ldots, 0$ & \\
\hline$b_{1}$ & 1 & 0 & \begin{tabular}{|l|lll|l}
$1, \ldots, 1$ & 1 & 0 & 0 & 0
\end{tabular} & $\overline{0, \ldots, 0}$ & \\
\hline$b_{2}$ & 1 & 1 & \begin{tabular}{|l|lll|l}
$1, \ldots, 1$ & 0 & 1 & 0 & 0
\end{tabular} & $0, \ldots, 0$ & \\
\hline \multirow[t]{2}{*}{$b_{3}$} & 1 & 0 & $|1, \ldots, 1| 00000$ & $0, \ldots, 0$ & \\
\hline & $y^{3, \ldots, 6}$ & $\bar{y}^{3, \ldots, 6}$ & $y^{1,2}, \omega^{5,6} \bar{y}^{1,2}, \bar{\omega}^{5,6}$ & \begin{tabular}{l|l}
6 & $\omega^{1, \ldots, 4}$
\end{tabular} & $\bar{\omega}^{1, \ldots, 4}$ \\
\hline 1 & $1, \ldots$ &, 1 & $1, \ldots, 1 \quad 1$ & 1 , &., 1 \\
\hline$S$ & $0, \ldots, 0$ & 0, . & $0, \ldots, 0 \quad 0, \ldots, 0$ & $0, \ldots, 0$ & $0, \ldots, 0$ \\
\hline$b_{1}$ & & & $0, \ldots, 0 \quad 0, \ldots, 0$ & 0, . & $0, \ldots, 0$ \\
\hline$b_{2}$ & 0, . & 0, . & $1, \ldots, 1 \quad 1, \ldots, 1$ & & $0, \ldots, 0$ \\
\hline$b_{3}$ & $0,$. & & $0, \ldots, 0 \quad 0, \ldots, 0$ & & $1, \ldots, 1$ \\
\hline
\end{tabular}

A suitable choice of GGSO phases that preserves spacetime supersymmetry is given by

$c\left(\begin{array}{l}b_{i} \\ b_{j}\end{array}\right)=c\left(\begin{array}{l}b_{i} \\ S\end{array}\right)=c\left(\begin{array}{l}\mathbf{1} \\ \mathbf{1}\end{array}\right)=-1$,

where all other GGSO projection coefficients are determined by modular invariance. The basis vector $S$ is the generator of spacetime supersymmetry. It merely corresponds to the Ramond vacuum of the worldsheet fermionic superpartners of the ten dimensional heterotic-string, and acts as a spectral flow operator that mixes between the spacetime fermionic and bosonic states.

The subset of basis vectors $\{\mathbf{1}, S\}$ produces a string vacuum with $N=4$ spacetime supersymmetry and $S O$ (44) gauge group. Adding the basis vectors $b_{1}$ and $b_{2}$ reduces the $N=4$ spacetime supersymmetry to $N=1$, where each of these vectors on its own reduces $N=4$ to $N=2$, and their combined action reduces $N=4$ to $N=1$. The characteristic of these two vectors is that their overlap with the basis vector $S$ yields $S \cdot b_{1,2}=2$. Thus, any additional vector that satisfies this overlap with the basis vector $S$ reduces the number of supersymmetries from $N=4$ to $N=2$. An example at hand is the basis vector $b_{3}$ of the NAHE set. However, the additional breaking induced by any additional basis vector with this property either preserves $N=1$ supersymmetry or reduces it further to $N=0$.

This type of breaking therefore produces a type of non supersymmetric string vacua that follow the chain $N=4 \rightarrow$ $N=2 \rightarrow N=1 \rightarrow N=0$. One characteristic of this type of spacetime supersymmetry breaking is that the breaking will a priori not be family universal. The reason is that the 
chiral families arise in the free fermionic models from the three sectors $b_{1}, b_{2}$ and $b_{3}$, and this type of breaking necessarily violates the cyclic permutation symmetry among the three sectors $b_{1}, b_{2}$ and $b_{3}$.

An example, where this cyclic permutation symmetry is instrumental in producing a family universal structure, is the supersymmetry breaking with a family universal anomalous $U(1)$ of $[60,61]$. The basis vectors $b_{1}, b_{2}$ and $b_{3}$ reduce the $S O(44)$ gauge symmetry to $S O(10) \times S O(6)^{3} \times E_{8}$. The gauge bosons that produce this gauge symmetry are obtained from the Neveu-Schwarz (NS) sector and the sector $Z=$ $1+b_{1}+b_{2}+b_{3}$, where the NS produces the vector bosons of $S O(10) \times S O(6)^{3} \times S O(16)$ and the sector $Z$ complements the $S O(16)$ group factor to $E_{8}$.

The NAHE set basis vectors $b_{1}, b_{2}$ and $b_{3}$ correspond to the three twisted sectors of the $Z_{2} \times Z_{2}$ toroidal orbifold. Each of these twisted sectors produces 16 multiplets in the spinorial 16 representation of $S O(10)$ to give a total of 48 chiral generations. The correspondence of the quasi-realistic free fermionic models with $Z_{2} \times Z_{2}$ orbifold has been amply discussed in the literature [62-69]. While the dictionary between specific models in the two approaches may be elusive, it is anticipated that for every model in one formalism there exists a representation in the alternative formalism and this should hold, at least for the $Z_{2} \times Z_{2}$ orbifolds, and higher order orbifolds may have a fermionic representation as well [70].

\subsubsection{Beyond the NAHE set}

The construction of the semi-realistic free fermionic models proceeds by adding three or four additional basis vectors to the NAHE set. The function of the additional basis vectors is to reduce the 48 spinorial $\mathbf{1 6}$ multiplets to three chiral generations, and at the same time to reduce the $S O(10)$ GUT symmetry to one of its subgroups:

(i) $S U(5) \times U(1)(\mathrm{FSU})$ [9];

(ii) $S U(3) \times S U(2) \times U(1)^{2}(\mathrm{SLM})[10,11]$;

(iii) $S O(6) \times S O(4)(\mathrm{PS})[12-14]$;

(iv) $S U(3) \times U(1) \times S U(2)^{2}(\mathrm{LRS})[15,16]$;

(v) $S U(4) \times S U(2) \times U(1)(\mathrm{SU} 421)[17]$.

The first four cases produced viable three generation models, whereas in the last case it was shown that phenomenologically viable models cannot be constructed [17,72,73]. The additional basis vectors may each preserve or break the $S O(10)$ symmetry. Basis vectors that preserve the $S O(10)$ symmetry are typically denoted by $b_{i}$ with $(i=4,5, \ldots)$, whereas those that break the $S O(10)$ symmetry are denoted by $\{\alpha, \beta, \gamma\}$. The overlap of the additional basis vectors with the supersymmetry generator basis vector $S$ determine the type of possible supersymmetry breaking. Thus, in the cases with $S \cdot b_{i}=2$ the pattern of supersymmetry breaking is similar to the spacetime supersymmetry breaking discussed above, namely it follows the chain $N=4 \rightarrow N=2 \rightarrow$ $N=1 \rightarrow N=0$.

An alternative is to use a basis vector with $S \cdot a_{i}=0$, where $a_{i}$ may, or may not, break the $S O(10)$ GUT symmetry. This type of supersymmetry breaking differs, however, from the one discussed above in that it induces the breaking pattern $N=4 \rightarrow N=0$. A general rule to construct vacua that preserve $N=1$ spacetime supersymmetry is to impose $c\left(\begin{array}{c}S \\ v_{i}\end{array}\right)=-\delta_{v_{i}}$, where $v_{i}$ is any basis vector [71]. Thus, relaxing this constraint would generically result in broken spacetime supersymmetry. Breaking spacetime supersymmetry with the additional basis vectors $a_{i}$ would generically also not affect the spectrum arising from the sectors $b_{i}$ that produce the chiral generations, but it may affect their superpartners as those are obtained from the sectors $S+b_{i}$. As we will show with an explicit example model in Sect. 4 , in this case the effect of the projection is to select different components of the underlying $N=4$ multiplets.

\subsubsection{The classification set}

In this paper our focus will be on non-supersymmetric NAHE-based free fermionic models. For completeness we discuss the construction of such models by using the classification methods of [18-21,23,46-48]. In this approach the set of basis vectors is fixed and a large number of string models, of the order of $10^{12}$ vacua, is spanned by enumerating the independent GGSO projection coefficients. In this manner large spaces of string models with $S O(10)$ [18,19], $S O(6) \times S O(4)$ [20,21], $S U(5) \times U(1)$ [23], and $S U(4) \times S U(2) \times U(1)[72,73]$, have been explored. A subset of basis vectors that respect the $S O(10)$ symmetry is given by the set of 12 basis vectors $V=\left\{v_{1}, v_{2}, \ldots, v_{12}\right\}$, where

$$
\begin{aligned}
v_{1}=1= & \left\{\psi^{\mu}, \chi^{1, \ldots, 6}, y^{1, \ldots, 6}, \omega^{1, \ldots, 6} \mid\right. \\
& \left.\bar{y}^{1, \ldots, 6}, \bar{\omega}^{1, \ldots, 6}, \bar{\eta}^{1,2,3}, \bar{\psi}^{1, \ldots, 5}, \bar{\phi}^{1, \ldots, 8}\right\}, \\
v_{2}=S= & \left\{\psi^{\mu}, \chi^{1, \ldots, 6}\right\}, \\
v_{2+i}=e_{i}= & \left\{y^{i}, \omega^{i} \mid \bar{y}^{i}, \bar{\omega}^{i}\right\}, i=1, \ldots, 6, \\
v_{9}= & b_{1}=\left\{\chi^{34}, \chi^{56}, y^{34}, y^{56} \mid \bar{y}^{34}, \bar{y}^{56}, \bar{\eta}^{1}, \bar{\psi}^{1, \ldots, 5}\right\}, \\
v_{10}= & b_{2}=\left\{\chi^{12}, \chi^{56}, y^{12}, y^{56} \mid \bar{y}^{12}, \bar{y}^{56}, \bar{\eta}^{2}, \bar{\psi}^{1, \ldots, 5}\right\}, \\
v_{11}= & z_{1}=\left\{\bar{\phi}^{1, \ldots, 4}\right\}, \\
v_{12}= & z_{2}=\left\{\bar{\phi}^{5, \ldots, 8}\right\} .
\end{aligned}
$$

Additional vectors are added to the set given in (2.12) to construct vacua with $S O(10)$ subgroups [20,21,23]. In the notation of Eq. (2.12) the worldsheet fermions appearing in the curly brackets have periodic boundary conditions, whereas all other worldsheet fermions are anti-periodic. The entries in the matrix of GGSO phases $c\left(\begin{array}{l}v_{i} \\ v_{j}\end{array}\right)$ with $i>j$ then span the space of string vacua. Additional constraints that are imposed on the string vacua, like the existence of spacetime 
supersymmetry leave 40 independent phases of the original 66. One can then resort to a complete [46-48] or statistical sampling ${ }^{1}$ of the total space $[18,19]$, and classify the models by their twisted matter spectrum. The classification is facilitated by expressing the GGSO projections in algebraic form $[18,19,46-48]$. The analysis of the entire spectrum of the string models is computerised and vacua with specific phenomenological characteristics can be fished our from the larger space of models.

In terms of spacetime supersymmetry breaking, as with the NAHE-set-based models the spacetime supersymmetry generator is the basis vector $S$. The subset $\{\mathbf{1}, S\}$ gives rise to $N=4$ spacetime supersymmetry, which is broken by $b_{1}$ and $b_{2}$ to $N=2$ spacetime supersymmetry and their combined action breaks $N=4 \rightarrow N=1$. As with the NAHE-based models imposing $c\left(\begin{array}{c}S \\ v_{i}\end{array}\right)=-\delta_{v_{i}}$ ensures the preservation of $N=1$ supersymmetry. Projecting the remaining supersymmetry in this model is obtained by relaxing this condition. Furthermore, the basis vectors $\left\{e_{i}, z_{1}, z_{2}\right\}$ satisfy $S \cdot e_{i}=0$ and $S \cdot z_{i}=0$. These basis vectors therefore act as projectors on the $S$ sector. These basis vectors can be used to project all the states from the $S$ sector and hence induce the breaking $N=4 \rightarrow N=0$ spacetime supersymmetry.

\section{Tachyons in the free fermionic semi-realistic models}

String models, in general, and heterotic-string models in particular, generically give rise to tachyonic states in their spectra. This can be seen from Eq. (2.6). Any sector that satisfies

$M_{L}^{2}<-\frac{1}{2}$ and $M_{R}^{2}<-1$

may produce tachyonic physical states. Tachyonic states can be obtained by acting on the vacuum with fermionic oscillators. They satisfy the level matching condition and survive all the GGSO projections. Their presence in the physical spectrum indicates the instability of the string vacuum. The existence of spacetime supersymmetry guarantees that all tachyonic states are projected out. The situation is altered if supersymmetry is broken to $N=0$ spacetime supersymmetry by projecting all the states from the $S$ sector. One then has to check in each model whether tachyonic states exist.

The existence of non-supersymmetric non-tachyonic string vacua has been known since the mid-1980s [24]. The gauge symmetry of this model is $S O(16) \times S O(16)$, and its non-perturbative extension was considered in [31]. In the free fermionic formalism the model is constructed by the set of boundary condition basis vectors $\{\mathbf{1}, S, X, I\}$ where $X=\left\{\bar{\psi}^{1, \ldots, 5}, \bar{\eta}^{1,2,3}\right\}$ and $I=\left\{\bar{\phi}^{1, \ldots, 8}\right\}$. In ten dimensions

1 We note that analysis of large sets of string vacua has also been carried out by other groups See e.g.: [27,74-85]. the choice of the GGSO phase $c\left(\begin{array}{c}X \\ I\end{array}\right)= \pm 1$ yields either the supersymmetric $E_{8} \times E_{8}$, or the non-supersymmetric $S O(16) \times S O(16)$, heterotic-string. This is necessarily the case in ten dimensions because the supersymmetry generator is given by $S=\mathbf{1}+X+I$ and therefore the projections on the three sectors are correlated. In the four dimensional models the same phase can be used to reduce the gauge symmetry from $E_{8} \times E_{8}$ to $S O(16) \times S O$ (16) without breaking supersymmetry. The same vacua can be constructed in the orbifold representation and can be connected by interpolations $[25,26]$. Hence, the supersymmetric and non-supersymmetric vacua exist on the boundary of the same moduli space.

Our interest in this paper is in the tachyonic states arising in the semi-realistic models. It is instructive to examine the case of the non-supersymmetric $S O(16) \times S O(16)$ model first. In the four dimensional model supersymmetry may be broken from $N=4 \rightarrow N=0$ by the $I$ or $X$ projections. The only sector that may produce tachyons in this model is the NS sector. The tachyonic states arising in this model are obtained by acting on the non-degenerate vacuum with a right-moving oscillator, and satisfy the level matching condition with $M_{L}^{2}=M_{R}^{2}=-1 / 2$. These tachyonic states are, however, projected out by the $S$ projection, which is given by

$\mathrm{e}^{i \pi S \cdot F_{N S}}|t\rangle_{N S}=\delta_{S}|t\rangle_{N S}$.

As there are no oscillators acting on the left-moving vacuum in the tachyonic untwisted state, and the basis vector $S$ is blind to the right-moving oscillators, the left-hand side of Eq. (3.2) is positive. On the other hand $\delta_{S}=-1$ because the spacetime fermions $\psi^{\mu}$ are periodic in $S$. The mismatch between the two sides of Eq. (3.2) entails that the untwisted NS tachyons are projected out. This argument extends to any free fermionic model that contains the basis vector $S$. We conclude that in any non-supersymmetric free fermionic that includes the $S$ sector the untwisted tachyons are always projected out, irrespective of the choice of the SUSY projecting phases.

The four dimensional $S O(16) \times S O(16)$ non-supersymmetric heterotic-string is therefore tachyon free. However, in this string vacuum the only sector that may give rise to tachyonic states is the untwisted sector. This is not the case in the semi-realistic free fermionic models. The three generation free fermionic models generically give rise to an abundance of sectors that may a priori give rise to tachyonic states. The reason is that the additional basis vectors that are required to reduce the number of families, break down the internal degrees of freedom into small units. This is exemplified by the set of vectors in Eq. (2.12), which is used as the basis for the classification of symmetric fermionic $Z_{2} \times Z_{2}$ orbifolds. The basis vectors $e_{i}$ as well as their $e_{i}+e_{j} i \neq j$ and $e_{i}+e_{j}+e_{k} i \neq j \neq k$ combinations may produce physical tachyonic states by acting on the 
non-degenerate vacuum with a right-moving NS fermionic oscillator; similarly the sectors $z_{\ell}, z_{\ell}+e_{i}, z_{\ell}+e_{i}+e_{j}, i \neq j$ $z_{\ell}+e_{i}+e_{j}+e_{k}, i \neq j \neq k$ may produce tachyonic states. In total there are therefore 123 sectors, in addition to the NS sector, that may produce tachyons in these models.

This renders futile a systematic classification of nonsupersymmetric non-tachyonic semi-realistic vacua along the lines of [20,21,23]. The situation in NAHE-based models is similar. Typical models contain an abundance of sectors that may a priori produce tachyons. Furthermore, models that utilise fractional boundary conditions may contain additional tachyon producing sectors in which a fermionic oscillator with rational boundary conditions may act on the non-degenerate vacuum. A detailed sector by sector analysis is therefore required. A systematic procedure to extract the tachyon-free vacua is provided by performing a $q$-expansion of the partition function [35]. However, this method loses the detailed information on the structure of the string spectrum. The construction of non-supersymmetric vacua with quasi-realistic features therefore requires a detailed model by model analysis. One may then envision the existence of models in which the number of tachyonic producing sectors is restricted.

The best case scenario would be a model in which the only tachyon producing sector is the NS sector. In this case we are guaranteed that tachyons do not exist in the physical spectrum. However, a model with this property has not been found to date. The next best case scenario is a model that gives rise only to one type of tachyon producing sectors. Existence of a model with this characteristic may depend on further detailed phenomenological properties of the string vacua. For example, we were not able to find such a model in the class of NAHE-based free fermionic models with reduced Higgs spectrum [54], whereas the class of left-right symmetric models $[15,16]$ did produce a model with the desired property. The set of boundary condition basis vectors, beyond the NAHE set, generating the string vacuum is given by

\begin{tabular}{|c|c|c|c|c|c|c|c|}
\hline & $\psi^{\mu}$ & $\chi^{12}$ & $\chi^{34}$ & $\chi^{56}$ & $\bar{\psi}^{1, \ldots, 5}$ & $\bar{\eta}^{1} \bar{\eta}^{2} \bar{\eta}^{3}$ & $\bar{\phi}^{1, \ldots, 8}$ \\
\hline$\alpha$ & 0 & 0 & 0 & 0 & 1111100 & \begin{tabular}{lll|}
0 & 0 & 0
\end{tabular} & 11110000 \\
\hline$\beta$ & 0 & 0 & 0 & 0 & 11100 & $\begin{array}{lll}0 & 0 & 0\end{array}$ & 11110000 \\
\hline$\gamma$ & 0 & 0 & 0 & 0 & $\begin{array}{ll}0 & 0\end{array}$ & $\frac{1}{2} \quad \frac{1}{2}$ & $\begin{array}{llllllll}0 & \frac{1}{2} & \frac{1}{2} & \frac{1}{2} & \frac{1}{2} & \frac{1}{2} & \frac{1}{2} & 0\end{array}$ \\
\hline
\end{tabular}

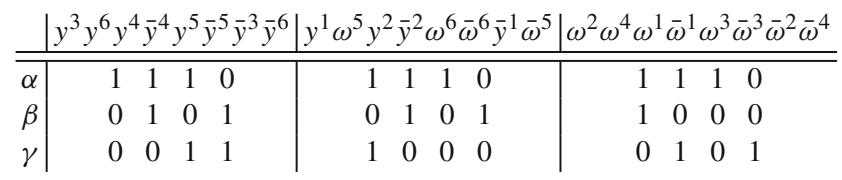

This model gives rise only to one type of tachyon producing sectors with

$\alpha_{L}^{2}=2 \& \alpha_{R}^{2}=6 \Rightarrow N_{R}=0$
The supersymmetric version of this model was presented in $[15,16]$ with the set of GGSO phases given by

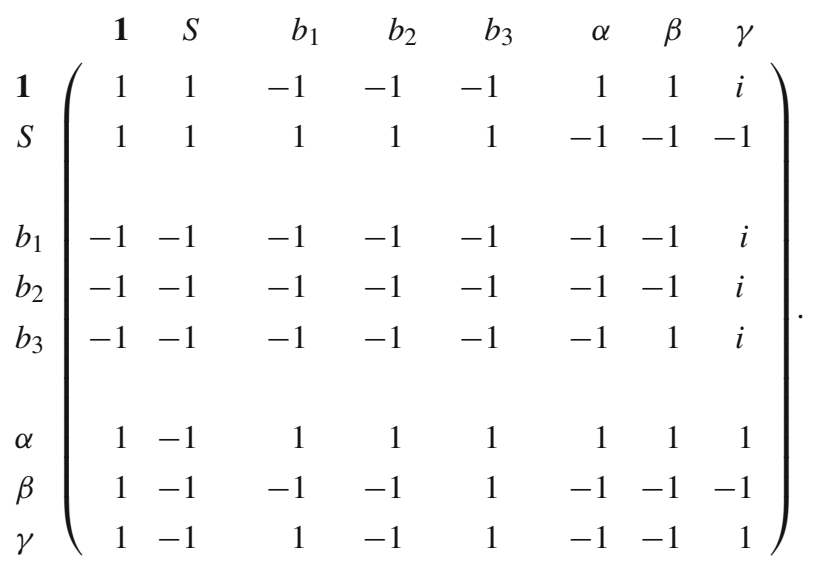

The full mass spectrum of this model together with the cubic level superpotential was presented in $[15,16]$. The modification

$c\left(\begin{array}{l}S \\ \alpha\end{array}\right)=-1 \rightarrow+1$ and $c\left(\begin{array}{l}S \\ \beta\end{array}\right)=-1 \rightarrow+1$

projects the remaining gravitino and induces $N=1 \rightarrow N=$ 0 . It can be checked that all the tachyonic states are projected out in this model. Furthermore, it can be verified that making the modification

$c\left(\begin{array}{l}S \\ \alpha\end{array}\right)=-1 \rightarrow+1$ and $c\left(\begin{array}{l}S \\ \beta\end{array}\right)=-1 \rightarrow-1$

i.e. modifying only $c\left(\begin{array}{l}S \\ \alpha\end{array}\right)$ but not $c\left(\begin{array}{l}S \\ \beta\end{array}\right)$ results in a model that contains tachyonic states. The reason is that in this model all the sectors that may produce tachyons appear with the combination $m(\alpha+\beta)$, where $m=0,1$. Hence, with the modification given by Eq. (3.6) the $S$-projection on the tachyonic sectors is the same as in the corresponding supersymmetry preserving choice given in Eq. (3.5), whereas with the modification given by (3.7) the $S$-projection in some sectors is modified in comparison to the supersymmetric model and some tachyonic states are not projected out. We note that the construction of tachyonic-free semi-realistic vacua is highly nontrivial. In the next section we discuss the tachyon-free model in some detail.

\section{An explicit tachyon-free model}

We consider the model defined by the set of basis vectors

$$
\begin{aligned}
1= & \left\{\psi^{\mu}, \chi^{1, \ldots, 6}, y^{1, \ldots, 6}, \omega^{1, \ldots, 6} \mid \bar{y}^{1, \ldots, 6}, \bar{\omega}^{1, \ldots, 6}, \bar{\eta}^{1,2,3},\right. \\
& \left.\bar{\psi}^{1, \ldots, 5}, \bar{\phi}^{1, \ldots, 8}\right\}, \\
S= & \left\{\psi^{\mu}, \chi^{1, \ldots, 6}\right\}, \\
b_{1}= & \left\{\psi^{\mu}, \chi^{1,2}, y^{3, \ldots, 6} \mid \bar{y}^{3, \ldots, 6}, \bar{\psi}^{1, \ldots, 5}, \bar{\eta}^{1}\right\},
\end{aligned}
$$




$$
\begin{aligned}
b_{2} & =\left\{\psi^{\mu}, \chi^{3,4}, y^{1,2}, \omega^{5,6} \mid \bar{y}^{1,2}, \bar{\omega}^{5,6}, \bar{\psi}^{1, \ldots, 5}, \bar{\eta}^{2}\right\}, \\
b_{3} & =\left\{\psi^{\mu}, \chi^{5,6}, \omega^{1, \ldots, 4} \mid \bar{\omega}^{1, \ldots, 4}, \bar{\psi}^{1, \ldots, 5}, \bar{\eta}^{3}\right\}, \\
b_{4} & =\alpha \\
& =\left\{y^{1, \ldots, 6}, \omega^{1, \ldots, 6} \mid \bar{\omega}^{1}, \bar{y}^{2}, \bar{\omega}^{3}, \bar{y}^{4,5}, \bar{\omega}^{6}, \bar{\psi}^{1,2,3}, \bar{\phi}^{1, \ldots, 4}\right\}, \\
b_{5} & =\beta \\
& =\left\{y^{2}, \omega^{2}, y^{4}, \omega^{4} \mid \bar{y}^{1, \ldots, 4}, \bar{\omega}^{5}, \bar{y}^{6}, \bar{\psi}^{1,2,3}, \bar{\phi}^{1, \ldots, 4}\right\}, \\
b_{6} & =\gamma \\
& =\left\{y^{1}, \omega^{1}, y^{5}, \omega^{5} \mid \bar{\omega}^{1,2}, \bar{y}^{3}, \bar{\omega}^{4}, \bar{y}^{5,6}, \bar{\psi}^{1,2,3}=\frac{1}{2}, \bar{\eta}^{1,2,3}\right. \\
& \left.=\frac{1}{2}, \bar{\phi}^{2, \ldots, 7}=\frac{1}{2}\right\},
\end{aligned}
$$

with the set of GGSO phases given by

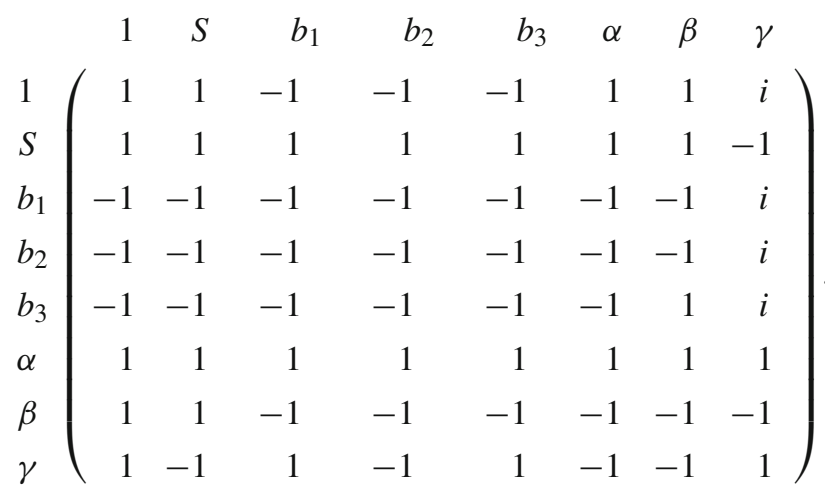

This is a three generation model, with one generation appearing in each of the twisted sectors $b_{1}, b_{2}$ and $b_{3}$. The full spectrum can be found in the tables of the appendix, with the exception of the gauge bosons which have been omitted in the interest of space. It is sufficient to state that the gauge group is

$$
\begin{aligned}
& \underbrace{S U(3)_{C} \times U(1)_{C} \times S U(2)_{L} \times S U(2)_{R} \times \prod_{i=1}^{6} U_{i}}_{\text {observable sector }} \\
& \times \underbrace{S U(3)_{H_{1}} \times S U(3)_{H_{2}} \times \prod_{j=7}^{10} U_{j}}_{\text {hidden sector }} .
\end{aligned}
$$

The notation for the table is the following: The first column describes if the states correspond to spacetime bosons or spacetime fermions and specifically for $b_{i}$ the type of particle. The second column is the name of the sector. The third column gives the dimensionality of the states under $S U(3)_{C} \times S U(2)_{L} \times S U(2)_{R}$ and the fourth the charges of the observable $U(1)$ s. Columns 5 and 6 describe the hidden sector. The only charges appearing in the table that do not have a self-evident name are:

$$
\begin{aligned}
Q_{C} & =Q_{\bar{\psi}^{1}}+Q_{\bar{\psi}^{2}}+Q_{\bar{\psi}^{3}}, \\
Q_{8} & =Q_{\bar{\phi}^{2}}+Q_{\bar{\phi}^{3}}+Q_{\bar{\phi}^{4}}, \\
Q_{9} & =Q_{\bar{\phi}^{5}}+Q_{\bar{\phi}^{6}}+Q_{\bar{\phi}^{7}} .
\end{aligned}
$$

To avoid writing fractional numbers all the charges in the table have been multiplied by 4 . Finally, for every state the CPT conjugate is also understood to be in the spectrum and has not been written explicitly. Lastly, we comment that the states contain discrete charges corresponding to the action of real fermions that are not shown in the table. For example, the first three states from the Neveu-Schwarz sector are obtained by acting on the vacuum with two right-moving real fermions and are neutral under the gauge symmetry of the model. The weak hypercharge in the model is given by

$U(1)_{Y}=\frac{1}{3} U(1)_{C}+T_{3_{R}}$,

where $T_{3_{R}}$ is the diagonal generator of $S U(2)_{R}$. The symmetry breaking to the Standard Model gauge group may be induced by a VEV for one of the Standard Model singlet scalar fields in the $(1,1,2)$ representation from the sectors $S+b_{j}$. This model exhibits many interesting features regarding supersymmetry. First of all, we observe that the model is manifestly non-supersymmetric. The gravitino and the gaugini are projected out and there is a clear mismatch between the number of states in the 0 and $S$ sectors. Furthermore, there are eight sectors with only scalars and the sectors that contain the would-be superpartners are massive. These are

$$
\begin{array}{ll}
\beta+\gamma, \quad \beta+3 \gamma, & \\
\alpha+\gamma, \quad \alpha+3 \gamma, & \\
1+b_{1}+b_{2}+b_{3}+\beta+\gamma, & 1+b_{1}+b_{2}+b_{3}+\beta+3 \gamma, \\
1+b_{1}+b_{2}+b_{3}+\alpha+\gamma, & 1+b_{1}+b_{2}+b_{3}+\alpha+3 \gamma .
\end{array}
$$

Such sectors would not remain in the spectrum in the supersymmetric choice of phases. The reason is that the spacetime supersymmetry generator in the supersymmetric model is the basis vector $S$, i.e. for a given sector $\rho \in \Xi$, the supersymmetric superpartners are obtained from the sector $S+\rho$. All the sectors in Eq. (4.2) have $(\rho)_{L}^{2}=4$, whereas $(S+\rho)_{L}^{2}=8$, i.e. in these sectors the would-be superpartners are massive. In the supersymmetric vacua the states from the sectors in Eq. (4.2) are necessarily projected out, as they break supersymmetry explicitly. However, once supersymmetry is broken they may appear in the spectrum, as is seen in our model. It is a highly non-trivial task to find a model with three generations in which sectors of these type, which only appear when supersymmetry is broken, contain no tachyons, but this model provides exactly such an example. We collectively refer to all the sectors mentioned in this paragraph as sectors in which supersymmetry is "badly broken".

On the other hand, there are (pairs of) sectors that are completely supersymmetric. This is due to the modification 
(3.6) not affecting the GGSO projections in any sectors where none of the vectors $S, \alpha$ or $\beta$ appear. Therefore such sectors will be identical to the corresponding sectors of the supersymmetric model. Nonetheless, for some of these sectors to remain supersymmetric as claimed above, the superpartners should be unchanged as well, or at least the effect must be (at most) a change in the $R$-charges of the superpartners. Sectors $b_{i}$ and $1+b_{i}+b_{j}+2 \gamma$ are of this type.

Finally, there are sectors that do not fit any of the above categories. In these sectors the number of bosons and fermions is the same, but on the other hand some of the gauge charges of these states are different which in principle prevents us from grouping them together into supermultiplets. Most of the sectors are of this type. We use the term sectors in which supersymmetry is "nicely broken" when referring to this case.

Thus, while supersymmetry is broken, some segments of the string vacuum still respect the underlying supersymmetric degeneracy. This is in accordance with the findings in [34], which showed that the partition function of string vacua with spontaneously broken supersymmetry can be divided into several orbits, some of which preserve the original supersymmetry.

Furthermore, we would like to comment in our model the fermionic states from the sectors $b_{1}, b_{2}$ and $b_{3}$, as well as the bosonic states from the NS sector, are not affected by the GGSO phases that project the gravitino and gaugini from the $S$ sector, and therefore break spacetime supersymmetry. Therefore, the untwisted scalar states of our non-supersymmetric model as well as the fermionic states from the sectors $b_{1}, b_{2}$ and $b_{3}$ are identical to those in the corresponding supersymmetric model. Consequently, the leading twisted-twisted-untwisted couplings in the nonsupersymmetric model, which are obtained by using the methods developed in [86-88], are identical to those of the supersymmetric model. The model generated by Eqs. $(3.3,3.5)$ contains electroweak doublet scalar representations from the twisted sectors that may be used as Higgs doublets. However, in this model the untwisted Higgs bi-doublets, which couple at leading order to the twisted sector states, are projected out and consequently the leading mass term which is identified with the top mass is absent. Other LRS $[15,16]$ models, as well as the FSU5 [9], PS [12-14] and SLM [3-5, 10,11] models, do contain the untwisted Higgs doublets and in those cases a leading top mass term is obtained.

It is also worth noting that even for non-supersymmetric models the cosmological constant can be exponentially suppressed. As discussed in [35], this can be achieved if the massless spectrum has an equal number of bosons and fermions (irrespectively of their charges). Even though our model is not of this type and will therefore have an unsuppressed vacuum energy, our construction hints at how one might go about achieving such a goal. It is clear, for example, that we do not have to worry about sectors that either respect supersymmetry or in which supersymmetry is nicely broken.

On the other hand, sectors that badly break supersymmetry will have to be carefully engineered. There are a few ways one might go about such a task. For example, one might entertain the idea that the addition of further basis vectors could project such sectors out of the spectrum. The biggest problem with this approach is that the removal of the gaugini from the $\mathrm{S}$ sector, even if some fermions transforming in a different from the adjoint representation are preserved, will create a mismatch of states in the S and NS sectors turning them into sectors that break supersymmetry badly; and it is impossible to project out the NS sector no matter what basis vectors are added. It is a priori possible that further basis vectors will remove exactly the correct number of bosons from the NS sector to match the remaining fermionic states in the $\mathrm{S}$ sector, but this method seems unnecessarily restricting.

An approach providing more freedom is to aim for an equality in the number of bosons and fermions not in each sector, but among different sectors. To cancel the surplus of bosons from the NS sector this would imply the existence of surviving fermionic states in different sectors, the bosonic counterpart of which has been projected out. The model presented in this section has an overall mismatch of bosonic and fermionic degrees of freedom and therefore does not satisfy this condition. Finding a semi-realistic model of suppressed cosmological constant appears to be very challenging, but it is of great interest as well and we hope to report on such constructions in a future publication.

\section{Split SUSY models}

In this section we briefly discuss string models with a split supersymmetry structure. The basic idea is to use two basis vectors to generate spacetime supersymmetry. We recall from Sect. 2 that in the semi-realistic free fermionic models the supersymmetry generators arise from the basis vector $S$. The aim in split supersymmetry string models is to construct two basis vectors that produce supersymmetry generators. A particular aim is then to construct models in which gaugini are obtained from one generator, whereas those of the second generator are projected out, as well as the scalar superpartners of the twisted matter fermionic states. Our construction proceeds by keeping our previous basis vector $S=\left\{\psi^{1,2}, \chi^{1, \ldots, 6}\right\} \equiv S_{1}$. A second supersymmetry generator is given by

$S_{2}=\left\{\psi^{1,2}, \chi^{1,2}, \omega^{3,4}, \omega^{5,6}\right\}$.

The basis vectors $b_{1}$ and $b_{2}$ of the NAHE-set equation (2.10) are added as well as the basis vectors $\mathbf{1}$ and $X$, which is used to project the supersymmetric generators from $S_{1}$, as discussed in Sect. 3. Shift basis vectors similar to the $e_{i}$ basis 
vectors of Eq. 2.12 can be added, and variations that include the basis vector $I$ of Sect. 2 . We consider the set of six basis vectors given by

$$
\begin{aligned}
v_{1}=1= & \left\{\psi^{\mu}, \chi^{1, \ldots, 6}, y^{1, \ldots, 6}, \omega^{1, \ldots, 6} \mid\right. \\
& \left.\bar{y}^{1, \ldots, 6}, \bar{\omega}^{1, \ldots, 6}, \bar{\eta}^{1,2,3}, \bar{\psi}^{1, \ldots, 5}, \bar{\phi}^{1, \ldots, 8}\right\}, \\
v_{2}= & S_{1}=\left\{\psi^{\mu}, \chi^{1, \ldots, 6}\right\}, \\
v_{3}=S_{2}= & \left\{\psi^{\mu}, \chi^{1,2}, \omega^{3, \ldots, 6}\right\}, \\
v_{4}=b_{1}= & \left\{\chi^{34}, \chi^{56}, y^{34}, y^{56} \mid \bar{y}^{34}, \bar{y}^{56}, \bar{\eta}^{1}, \bar{\psi}^{1, \ldots, 5}\right\}, \\
v_{5}=X= & \left\{\bar{\eta}^{1,2,3}, \bar{\psi}^{1, \ldots, 5}\right\}, \\
v_{6}=I= & \left\{\bar{\phi}^{1, \ldots, 8}\right\} .
\end{aligned}
$$

\begin{tabular}{|c|c|c|c|c|c|c|}
\hline & 1 & $S_{1}$ & $S_{2}$ & $b_{1}$ & $X$ & $I$ \\
\hline & $(-1$ & -1 & -1 & -1 & -1 & -1 \\
\hline$S_{1}$ & -1 & -1 & -1 & 1 & 1 & 1 \\
\hline$S_{2}$ & -1 & 1 & -1 & 1 & -1 & -1 \\
\hline & -1 & -1 & -1 & -1 & 1 & -1 \\
\hline & -1 & 1 & -1 & -1 & 1 & -1 \\
\hline & -1 & 1 & -1 & -1 & -1 & \\
\hline
\end{tabular}

with the set of GGSO phases given by

In this model the NS sector is the only sector that produces spacetime vector bosons. Hence the gauge symmetry in four dimensions is $S O(8) \times S O(4) \times S O(4) \times S O(12) \times S O(16)$. The sector $b_{1}$ gives rise to spacetime fermions in the spinor and anti-spinor representations of $S O(12)$. The supersymmetry generators of $S_{1}$ are projected out, whereas the gaugini from $S_{2}$ are retained. The model retains the scalar superpartners from the sector $S_{2}+b_{1}$, and projects those from the sector $S_{1}+b_{1}$. Our general aim in the construction of models with split supersymmetry is to construct models that retain the gaugini and spacetime fermions from $S_{2}$ and $b_{1}$, while projecting the gaugini (and hence the gravitini) from $S_{1}$, as well as the superpartners from the sectors $S_{1}+b_{1}$ and $S_{2}+b_{1}$. However, variations of the model in Eqs. $(5.2,5.3)$, including adding the $e_{i}$ projectors of Eq. (2.12) did not yield the desired result. The models in which supersymmetry is entirely broken, i.e. those in which the supersymmetry generators from $S_{1}$ as well as $S_{2}$ are projected out, typically contain tachyons. We then face similar situation to the one discussed in Sect. 2.1.3.

\section{Conclusions}

The observation of a scalar resonance compatible with the electroweak Higgs doublet reinforces the hypothesis that the Standard Model provides a viable parameterisation of all sub-atomic data up to the Planck scale. Synthesis of gravity with the sub-atomic interactions necessitates a departure from the local point particle idealisation of quantum field theories, which underly the Standard Model. The most developed framework to explore the gauge-gravity augmentation is offered by string theory. Detailed phenomenological models that incorporate the salient feature of the Standard Model have been constructed. These detailed phenomenological constructions contain a new symmetry, i.e. $N=1$ spacetime supersymmetry, which has not been observed to date in experiments.

A vital question is therefore to explore the consequences of breaking spacetime supersymmetry directly at the string scale. A generic feature of non-supersymmetric string vacua is the existence of tachyonic states in the physical spectrum. Non-supersymmetric string vacua, such as the $S O(16) \times$ $S O(16)$ heterotic-string in ten dimensions, do not contain tachyonic states, but are typically connected in the moduli space to supersymmetric vacua, and tend to have large moduli states and group factors. More realistic constructions on the other hand, typically have reduced moduli spaces and contain more sectors that may a priori give rise to tachyons.

It is therefore important to examine the structure of nonsupersymmetric string vacua in more realistic setting. In this paper we undertook this task. We have shown that while generically the quasi-realistic non-supersymmetric vacua do contain tachyons, there also exist examples in which all the tachyonic states are projected out by the GGSO projections. Furthermore, given that the moduli spaces of the quasi-realistic constructions may be much reduced [53,54], one may entertain the possibility that the tachyon-free nonsupersymmetric quasi-realistic vacua may not be connected to supersymmetric solutions.

We have shown with a concrete example that the nonsupersymmetric quasi-realistic vacuum may retain some of the structure of the corresponding supersymmetric solution. This demonstrates that even though supersymmetry may be broken directly at the string level, the effective spectrum of the string vacuum, as well as its low energy effective field theory, may still exhibit properties that are similar to those of the corresponding supersymmetric solutions, e.g. the existence of scalar replications of the chiral generations.

Another interesting point to note is the existence of an anomalous $U(1)$ symmetry in the model. The anomalous $U(1)$ is cancelled by the Green-Schwarz-Dine-SeibergWitten mechanism [89,90], but gives rise to a tadpole diagram at one-loop order in string perturbation theory [91], which reflects the instability of the string vacuum. The mismatch between the fermionic and bosonic states at different mass levels gives rise to a non-vanishing vacuum energy, which similarly gives rise to a tadpole diagram, indicating the instability of the string vacuum. We may contemplate the possibility of employing one against the other so that they conspire to cancel. The anomalous $U(1)$ contribution is proportional to the trace over the massless fermionic states and the sign can be altered by the GGSO projections [91,92]. It 
is proportional to the gauge coupling and consequently only depends on the dilaton moduli. On the other hand, the vacuum amplitude contribution depends on other moduli [35], and it may be tuned to obtain cancellation of the two contributions. In general, other background fields will be affected by the shift of the vacuum, and to demonstrate the existence of a stable vacuum one would need to solve the set of equations affecting those fields in the shifted vacuum. However, in this regard the same constraints would apply in the case of the supersymmetric vacua, where the Fayet-Iliopoulos term $[90,93]$, which is generated from the anomalous $U(1)$ tadpole diagram [90,91], is cancelled by assigning VEVs to some massless scalar fields, along flat supersymmetric directions. It would appear therefore that this shift of the vacuum is either legitimate, or illegitimate, in both cases. We therefore propose that the non-supersymmetric non-tachyonic string vacua should be considered on equal footing to the supersymmetric examples.
Acknowledgments We would like to thank Steve Abel, Ignatios Antoniadis, Costas Kounnas, Thomas Mohaupt, Marios Petropoulos and John Rizos for discussions. A.E.F. would like to thank the Institute for Advanced Study for hospitality. A.E.F. is supported in part by STFC under contract ST/L000431/1. P.A. acknowledges support from the Hellenic State Scholarship Foundation (IKY).

Open Access This article is distributed under the terms of the Creative Commons Attribution 4.0 International License (http://creativecomm ons.org/licenses/by/4.0/), which permits unrestricted use, distribution, and reproduction in any medium, provided you give appropriate credit to the original author(s) and the source, provide a link to the Creative Commons license, and indicate if changes were made.

Funded by SCOAP ${ }^{3}$.

\section{A The spectrum of the model in Sect. 4}

See Tables 1, 2, 3, 4, 5, 6, 7, 8 and 9 .

Table 1 The untwisted Neveu-Schwarz sector matter states and charges

\begin{tabular}{|c|c|c|c|c|c|c|c|c|c|c|c|c|c|c|}
\hline$F$ & SEC & $(C ; L ; R)$ & $Q_{C}$ & $Q_{\bar{\eta}^{1}}$ & $Q_{\bar{\eta}^{2}}$ & $Q_{\bar{\eta}^{3}}$ & $Q_{\bar{y}^{3}, 6}$ & $Q_{\bar{y}^{1} \bar{w}^{5}}$ & $Q_{\bar{w}^{2,4}}$ & $S U(3)_{H_{1,2}}$ & $Q_{\bar{\Phi}^{1}}$ & $Q_{8}$ & $Q_{9}$ & $Q_{\bar{\Phi}^{8}}$ \\
\hline \multirow[t]{9}{*}{ b } & \multirow[t]{9}{*}{ NS } & $(1,1,1)$ & 0 & 0 & 0 & 0 & 0 & 0 & 0 & $(1,1)$ & 0 & 0 & 0 & 0 \\
\hline & & $(1,1,1)$ & 0 & 0 & 0 & 0 & 0 & 0 & 0 & $(1,1)$ & 0 & 0 & 0 & 0 \\
\hline & & $(1,1,1)$ & 0 & 0 & 0 & 0 & 0 & 0 & 0 & $(1,1)$ & 0 & 0 & 0 & 0 \\
\hline & & $(1,1,1)$ & 0 & -4 & 4 & 0 & 0 & 0 & 0 & $(1,1)$ & 0 & 0 & 0 & 0 \\
\hline & & $(1,1,1)$ & 0 & 4 & -4 & 0 & 0 & 0 & 0 & $(1,1)$ & 0 & 0 & 0 & 0 \\
\hline & & $(1,1,1)$ & 0 & 0 & -4 & 4 & 0 & 0 & 0 & $(1,1)$ & 0 & 0 & 0 & 0 \\
\hline & & $(1,1,1)$ & 0 & 0 & 4 & -4 & 0 & 0 & 0 & $(1,1)$ & 0 & 0 & 0 & 0 \\
\hline & & $(1,1,1)$ & 0 & -4 & 0 & 4 & 0 & 0 & 0 & $(1,1)$ & 0 & 0 & 0 & 0 \\
\hline & & $(1,1,1)$ & 0 & 4 & 0 & -4 & 0 & 0 & 0 & $(1,1)$ & 0 & 0 & 0 & 0 \\
\hline
\end{tabular}

Table 2 The untwisted $S$ sector matter states and charges

\begin{tabular}{|c|c|c|c|c|c|c|c|c|c|c|c|c|c|c|}
\hline$F$ & SEC & $(C ; L ; R)$ & $Q_{C}$ & $Q_{\bar{\eta}^{1}}$ & $Q_{\bar{\eta}^{2}}$ & $Q_{\bar{\eta}^{3}}$ & $Q_{\bar{y}^{3,6}}$ & $Q_{\bar{y}^{1} \bar{w}^{5}}$ & $Q_{\bar{w}^{2,4}}$ & $S U(3)_{H_{1,2}}$ & $Q_{\bar{\Phi}^{1}}$ & $Q_{8}$ & $Q_{9}$ & $Q_{\bar{\Phi}^{8}}$ \\
\hline \multirow[t]{11}{*}{$\mathrm{f}$} & \multirow[t]{11}{*}{$S$} & $(1,1,1)$ & 0 & 0 & 0 & 0 & 0 & 0 & 0 & $(3, \overline{3})$ & 0 & -4 & 4 & 0 \\
\hline & & $(1,1,1)$ & 0 & 0 & 0 & 0 & 0 & 0 & 0 & $(3, \overline{3})$ & 0 & 4 & -4 & 0 \\
\hline & & $(1,1,1)$ & 0 & 0 & 0 & 0 & 0 & 0 & 0 & $(1,1)$ & 4 & 0 & 0 & 4 \\
\hline & & $(1,1,1)$ & 0 & 0 & 0 & 0 & 0 & 0 & 0 & $(1,1)$ & 4 & 0 & 0 & -4 \\
\hline & & $(1,1,1)$ & 0 & 0 & 0 & 0 & 0 & 0 & 0 & $(1,1)$ & -4 & 0 & 0 & 4 \\
\hline & & $(1,1,1)$ & 0 & 0 & 0 & 0 & 0 & 0 & 0 & $(1,1)$ & -4 & 0 & 0 & -4 \\
\hline & & $(3,1,1)$ & -4 & 4 & 0 & 0 & 0 & 0 & 0 & $(1,1)$ & 0 & 0 & 0 & 0 \\
\hline & & $(\overline{3}, 1,1)$ & 4 & -4 & 0 & 0 & 0 & 0 & 0 & $(1,1)$ & 0 & 0 & 0 & 0 \\
\hline & & $(3,1,1)$ & -4 & 0 & 4 & 0 & 0 & 0 & 0 & $(1,1)$ & 0 & 0 & 0 & 0 \\
\hline & & $(\overline{3}, 1,1)$ & 4 & 0 & -4 & 0 & 0 & 0 & 0 & $(1,1)$ & 0 & 0 & 0 & 0 \\
\hline & & $(3,1,1)$ & -4 & 0 & 0 & 4 & 0 & 0 & 0 & $(1,1)$ & 0 & 0 & 0 & 0 \\
\hline
\end{tabular}


Table 2 continued

\begin{tabular}{|c|c|c|c|c|c|c|c|c|c|c|c|c|c|c|}
\hline$F$ & SEC & $(C ; L ; R)$ & $Q_{C}$ & $Q_{\bar{\eta}^{1}}$ & $Q_{\bar{\eta}^{2}}$ & $Q_{\bar{\eta}^{3}}$ & $Q_{\bar{y}^{3,6}}$ & $Q_{\bar{y}^{1} \bar{w}^{5}}$ & $Q_{\bar{w}^{2,4}}$ & $S U(3)_{H_{1,2}}$ & $Q_{\bar{\Phi}^{1}}$ & $Q_{8}$ & $Q_{9}$ & $Q_{\bar{\Phi}^{8}}$ \\
\hline & & $(\overline{3}, 1,1)$ & 4 & 0 & 0 & -4 & 0 & 0 & 0 & $(1,1)$ & 0 & 0 & 0 & 0 \\
\hline & & $(1,2,2)$ & 0 & 0 & 0 & 0 & 0 & 0 & 0 & $(1,1)$ & 0 & 0 & 0 & 0 \\
\hline & & $(1,2,2)$ & 0 & 0 & 0 & 0 & 0 & 0 & 0 & $(1,1)$ & 0 & 0 & 0 & 0 \\
\hline & & $(1,1,1)$ & 0 & 0 & 0 & 0 & 4 & 0 & 0 & $(1,1)$ & 0 & 0 & 0 & 0 \\
\hline & & $(1,1,1)$ & 0 & 0 & 0 & 0 & -4 & 0 & 0 & $(1,1)$ & 0 & 0 & 0 & 0 \\
\hline & & $(1,1,1)$ & 0 & 0 & 0 & 0 & 0 & 4 & 0 & $(1,1)$ & 0 & 0 & 0 & 0 \\
\hline & & $(1,1,1)$ & 0 & 0 & 0 & 0 & 0 & -4 & 0 & $(1,1)$ & 0 & 0 & 0 & 0 \\
\hline & & $(1,1,1)$ & 0 & 0 & 0 & 0 & 4 & 0 & 0 & $(1,1)$ & 0 & 0 & 0 & 0 \\
\hline & & $(1,1,1)$ & 0 & 0 & 0 & 0 & -4 & 0 & 0 & $(1,1)$ & 0 & 0 & 0 & 0 \\
\hline & & $(1,1,1)$ & 0 & 0 & 0 & 0 & 0 & 4 & 0 & $(1,1)$ & 0 & 0 & 0 & 0 \\
\hline & & $(1,1,1)$ & 0 & 0 & 0 & 0 & 0 & -4 & 0 & $(1,1)$ & 0 & 0 & 0 & 0 \\
\hline
\end{tabular}

Table 3 The observable matter sectors. All sectors, fermionic and bosonic, have CPT conjugates which are not displayed

\begin{tabular}{|c|c|c|c|c|c|c|c|c|c|c|c|c|c|c|}
\hline$F$ & SEC & $(C ; L ; R)$ & $Q_{C}$ & $Q_{\bar{\eta}^{1}}$ & $Q_{\bar{\eta}^{2}}$ & $Q_{\bar{\eta}^{3}}$ & $Q_{\bar{y}^{3,6}}$ & $Q_{\bar{y}^{1} \bar{w}^{5}}$ & $Q_{\bar{w}^{2,4}}$ & $S U(3)_{H_{1,2}}$ & $Q_{\bar{\Phi}^{1}}$ & $Q_{8}$ & $Q_{9}$ & $Q_{\bar{\Phi}^{8}}$ \\
\hline$Q_{L_{1}}$ & $b_{1}$ & $(3,2,1)$ & 2 & 2 & 0 & 0 & -2 & 0 & 0 & $(1,1)$ & 0 & 0 & 0 & 0 \\
\hline$Q_{R_{1}}$ & & $(\overline{3}, 1,2)$ & -2 & -2 & 0 & 0 & -2 & 0 & 0 & $(1,1)$ & 0 & 0 & 0 & 0 \\
\hline$L_{L_{1}}$ & & $(1,2,1)$ & -6 & 2 & 0 & 0 & -2 & 0 & 0 & $(1,1)$ & 0 & 0 & 0 & 0 \\
\hline$L_{R_{1}}$ & & $(1,1,2)$ & 6 & -2 & 0 & 0 & -2 & 0 & 0 & $(1,1)$ & 0 & 0 & 0 & 0 \\
\hline \multirow[t]{4}{*}{$\mathrm{b}$} & $S+b_{1}$ & $(3,1,2)$ & 2 & 2 & 0 & 0 & -2 & 0 & 0 & $(1,1)$ & 0 & 0 & 0 & 0 \\
\hline & & $(\overline{3}, 2,1)$ & -2 & -2 & 0 & 0 & -2 & 0 & 0 & $(1,1)$ & 0 & 0 & 0 & 0 \\
\hline & & $(1,2,1)$ & 6 & -2 & 0 & 0 & -2 & 0 & 0 & $(1,1)$ & 0 & 0 & 0 & 0 \\
\hline & & $(1,1,2)$ & -6 & 2 & 0 & 0 & -2 & 0 & 0 & $(1,1)$ & 0 & 0 & 0 & 0 \\
\hline$Q_{L_{2}}$ & $b_{2}$ & $(3,2,1)$ & 2 & 0 & 2 & 0 & 0 & -2 & 0 & $(1,1)$ & 0 & 0 & 0 & 0 \\
\hline$Q_{R_{2}}$ & & $(\overline{3}, 1,2)$ & -2 & 0 & -2 & 0 & 0 & -2 & 0 & $(1,1)$ & 0 & 0 & 0 & 0 \\
\hline$L_{L_{2}}$ & & $(1,2,1)$ & -6 & 0 & 2 & 0 & 0 & -2 & 0 & $(1,1)$ & 0 & 0 & 0 & 0 \\
\hline$L_{R_{2}}$ & & $(1,1,2)$ & 6 & 0 & -2 & 0 & 0 & -2 & 0 & $(1,1)$ & 0 & 0 & 0 & 0 \\
\hline \multirow[t]{4}{*}{$\mathrm{b}$} & $S+b_{2}$ & $(3,1,2)$ & 2 & 0 & 2 & 0 & 0 & -2 & 0 & $(1,1)$ & 0 & 0 & 0 & 0 \\
\hline & & $(\overline{3}, 2,1)$ & -2 & 0 & -2 & 0 & 0 & -2 & 0 & $(1,1)$ & 0 & 0 & 0 & 0 \\
\hline & & $(1,2,1)$ & 6 & 0 & -2 & 0 & 0 & -2 & 0 & $(1,1)$ & 0 & 0 & 0 & 0 \\
\hline & & $(1,1,2)$ & -6 & 0 & 2 & 0 & 0 & -2 & 0 & $(1,1)$ & 0 & 0 & 0 & 0 \\
\hline$Q_{L_{3}}$ & $b_{3}$ & $(3,2,1)$ & 2 & 0 & 0 & 2 & 0 & 0 & -2 & $(1,1)$ & 0 & 0 & 0 & 0 \\
\hline$Q_{R_{3}}$ & & $(\overline{3}, 1,2)$ & -2 & 0 & 0 & -2 & 0 & 0 & -2 & $(1,1)$ & 0 & 0 & 0 & 0 \\
\hline$L_{L_{3}}$ & & $(1,2,1)$ & -6 & 0 & 0 & 2 & 0 & 0 & -2 & $(1,1)$ & 0 & 0 & 0 & 0 \\
\hline$L_{R_{3}}$ & & $(1,1,2)$ & 6 & 0 & 0 & -2 & 0 & 0 & -2 & $(1,1)$ & 0 & 0 & 0 & 0 \\
\hline \multirow[t]{4}{*}{$\mathrm{b}$} & $S+b_{3}$ & $(3,1,2)$ & 2 & 0 & 0 & 2 & 0 & 0 & -2 & $(1,1)$ & 0 & 0 & 0 & 0 \\
\hline & & $(\overline{3}, 2,1)$ & -2 & 0 & 0 & -2 & 0 & 0 & -2 & $(1,1)$ & 0 & 0 & 0 & 0 \\
\hline & & $(1,2,1)$ & 6 & 0 & 0 & -2 & 0 & 0 & -2 & $(1,1)$ & 0 & 0 & 0 & 0 \\
\hline & & $(1,1,2)$ & -6 & 0 & 0 & 2 & 0 & 0 & -2 & $(1,1)$ & 0 & 0 & 0 & 0 \\
\hline
\end{tabular}


Table 4 Vector-like $S O(10)$ singlet states. All sectors, fermionic and bosonic, have CPT conjugates which are not displayed

\begin{tabular}{|c|c|c|c|c|c|c|c|c|c|c|c|c|c|c|}
\hline$F$ & SEC & $(C ; L ; R)$ & $Q_{C}$ & $Q_{\bar{\eta}^{1}}$ & $Q_{\bar{\eta}^{2}}$ & $Q_{\bar{\eta}^{3}}$ & $Q_{\bar{y} 3,6}$ & $Q_{\bar{y}^{1} \bar{w}^{5}}$ & $Q_{\bar{w}^{2,4}}$ & $S U(3)_{H_{1,2}}$ & $Q_{\bar{\Phi}^{1}}$ & $Q_{8}$ & $Q_{9}$ & $Q_{\bar{\Phi}^{8}}$ \\
\hline \multirow[t]{8}{*}{$f$} & $S+$ & $(1,1,1)$ & 0 & 2 & -2 & 0 & 0 & 0 & 0 & $(1,1)$ & 0 & 0 & 0 & 4 \\
\hline & $b_{1}+b_{2}$ & $(1,1,1)$ & 0 & 2 & -2 & 0 & 0 & 0 & 0 & $(1,1)$ & 0 & 0 & 0 & -4 \\
\hline & $+\alpha+\beta$ & $(1,1,1)$ & 0 & -2 & 2 & 0 & 0 & 0 & 0 & $(1,1)$ & 0 & 0 & 0 & 4 \\
\hline & & $(1,1,1)$ & 0 & -2 & 2 & 0 & 0 & 0 & 0 & $(1,1)$ & 0 & 0 & 0 & -4 \\
\hline & & $(1,1,1)$ & 0 & 2 & 2 & 0 & 0 & 0 & 0 & $(3,1)$ & 0 & 4 & 0 & 0 \\
\hline & & $(1,1,1)$ & 0 & -2 & -2 & 0 & 0 & 0 & 0 & $(3,1)$ & 0 & 4 & 0 & 0 \\
\hline & & $(1,1,1)$ & 0 & 2 & 2 & 0 & 0 & 0 & 0 & $(\overline{3}, 1)$ & 0 & -4 & 0 & 0 \\
\hline & & $(1,1,1)$ & 0 & -2 & -2 & 0 & 0 & 0 & 0 & $(\overline{3}, 1)$ & 0 & -4 & 0 & 0 \\
\hline \multirow[t]{8}{*}{$\mathrm{b}$} & $b_{1}+b_{2}$ & $(1,1,1)$ & 0 & 2 & -2 & 0 & 0 & 0 & 0 & $(1,1)$ & 4 & 0 & 0 & 0 \\
\hline & $+\alpha+\beta$ & $(1,1,1)$ & 0 & 2 & -2 & 0 & 0 & 0 & 0 & $(1,1)$ & -4 & 0 & 0 & 0 \\
\hline & & $(1,1,1)$ & 0 & -2 & 2 & 0 & 0 & 0 & 0 & $(1,1)$ & 4 & 0 & 0 & 0 \\
\hline & & $(1,1,1)$ & 0 & -2 & 2 & 0 & 0 & 0 & 0 & $(1,1)$ & -4 & 0 & 0 & 0 \\
\hline & & $(1,1,1)$ & 0 & 2 & 2 & 0 & 0 & 0 & 0 & $(1,3)$ & 0 & 0 & 4 & 0 \\
\hline & & $(1,1,1)$ & 0 & -2 & -2 & 0 & 0 & 0 & 0 & $(1,3)$ & 0 & 0 & 4 & 0 \\
\hline & & $(1,1,1)$ & 0 & 2 & 2 & 0 & 0 & 0 & 0 & $(1, \overline{3})$ & 0 & 0 & -4 & 0 \\
\hline & & $(1,1,1)$ & 0 & -2 & -2 & 0 & 0 & 0 & 0 & $(1, \overline{3})$ & 0 & 0 & -4 & 0 \\
\hline
\end{tabular}

Table 5 Vector-like $S O(10)$ singlet states. All sectors, fermionic and bosonic, have CPT conjugates which are not displayed

\begin{tabular}{|c|c|c|c|c|c|c|c|c|c|c|c|c|c|c|}
\hline$F$ & SEC & $(C ; L ; R)$ & $Q_{C}$ & $Q_{\bar{\eta}^{1}}$ & $Q_{\bar{\eta}^{2}}$ & $Q_{\bar{\eta}^{3}}$ & $Q_{\bar{y}^{3,6}}$ & $Q_{\bar{y}^{1} \bar{w}^{5}}$ & $Q_{\bar{w}^{2,4}}$ & $S U(3)_{H_{1,2}}$ & $Q_{\bar{\Phi}^{1}}$ & $Q_{8}$ & $Q_{9}$ & $Q_{\bar{\Phi}^{8}}$ \\
\hline \multirow[t]{4}{*}{$\mathrm{f}$} & $S+$ & $(1,1,1)$ & 0 & -2 & 0 & 0 & 0 & -2 & -2 & $(1,1)$ & 0 & 6 & 0 & 2 \\
\hline & $1+b_{1}$ & $(1,1,1)$ & 0 & 2 & 0 & 0 & 0 & -2 & -2 & $(1,1)$ & 0 & -6 & 0 & -2 \\
\hline & \multirow[t]{2}{*}{$+\alpha+2 \gamma$} & $(1,1,1)$ & 0 & 2 & 0 & 0 & 0 & -2 & -2 & $(3,1)$ & 0 & 2 & 0 & -2 \\
\hline & & $(1,1,1)$ & 0 & -2 & 0 & 0 & 0 & -2 & -2 & $(\overline{3}, 1)$ & 0 & -2 & 0 & 2 \\
\hline \multirow[t]{4}{*}{$\mathrm{b}$} & $1+b_{1}$ & $(1,1,1)$ & 0 & -2 & 0 & 0 & 0 & 2 & -2 & $(1,1)$ & 0 & 6 & 0 & 2 \\
\hline & \multirow{3}{*}{$+\alpha+2 \gamma$} & $(1,1,1)$ & 0 & 2 & 0 & 0 & 0 & 2 & -2 & $(1,1)$ & 0 & -6 & 0 & -2 \\
\hline & & $(1,1,1)$ & 0 & 2 & 0 & 0 & 0 & 2 & -2 & $(3,1)$ & 0 & 2 & 0 & -2 \\
\hline & & $(1,1,1)$ & 0 & -2 & 0 & 0 & 0 & 2 & -2 & $(\overline{3}, 1)$ & 0 & -2 & 0 & 2 \\
\hline \multirow[t]{4}{*}{$\mathrm{f}$} & $S+$ & $(1,1,1)$ & 0 & 0 & -2 & 0 & -2 & 0 & -2 & $(1,1)$ & 0 & 6 & 0 & 2 \\
\hline & $1+b_{2}$ & $(1,1,1)$ & 0 & 0 & 2 & 0 & -2 & 0 & -2 & $(1,1)$ & 0 & -6 & 0 & -2 \\
\hline & \multirow[t]{2}{*}{$+\alpha+2 \gamma$} & $(1,1,1)$ & 0 & 0 & 2 & 0 & -2 & 0 & -2 & $(3,1)$ & 0 & 2 & 0 & -2 \\
\hline & & $(1,1,1)$ & 0 & 0 & -2 & 0 & -2 & 0 & -2 & $(\overline{3}, 1)$ & 0 & -2 & 0 & 2 \\
\hline \multirow[t]{4}{*}{$\mathrm{b}$} & $1+b_{2}$ & $(1,1,1)$ & 0 & 0 & -2 & 0 & 2 & 0 & -2 & $(1,1)$ & 0 & 6 & 0 & 2 \\
\hline & \multirow[t]{3}{*}{$+\alpha+2 \gamma$} & $(1,1,1)$ & 0 & 0 & 2 & 0 & 2 & 0 & -2 & $(1,1)$ & 0 & -6 & 0 & -2 \\
\hline & & $(1,1,1)$ & 0 & 0 & 2 & 0 & 2 & 0 & -2 & $(3,1)$ & 0 & 2 & 0 & -2 \\
\hline & & $(1,1,1)$ & 0 & 0 & -2 & 0 & 2 & 0 & -2 & $(\overline{3}, 1)$ & 0 & -2 & 0 & 2 \\
\hline \multirow[t]{4}{*}{$\mathrm{f}$} & $S+$ & $(1,1,1)$ & 0 & 0 & -2 & 0 & -2 & 0 & -2 & $(1,1)$ & -2 & 0 & 6 & 0 \\
\hline & $b_{1}+b_{3}$ & $(1,1,1)$ & 0 & 0 & 2 & 0 & -2 & 0 & -2 & $(1,1)$ & 2 & 0 & -6 & 0 \\
\hline & \multirow[t]{2}{*}{$+\alpha+2 \gamma$} & $(1,1,1)$ & 0 & 0 & 2 & 0 & -2 & 0 & -2 & $(1,3)$ & 2 & 0 & 2 & 0 \\
\hline & & $(1,1,1)$ & 0 & 0 & -2 & 0 & -2 & 0 & -2 & $(1, \overline{3})$ & -2 & 0 & -2 & 0 \\
\hline \multirow[t]{4}{*}{$\mathrm{b}$} & $b_{1}+b_{3}$ & $(1,1,1)$ & 0 & 0 & -2 & 0 & 2 & 0 & -2 & $(1,1)$ & -2 & 0 & 6 & 0 \\
\hline & \multirow[t]{3}{*}{$+\alpha+2 \gamma$} & $(1,1,1)$ & 0 & 0 & 2 & 0 & 2 & 0 & -2 & $(1,1)$ & 2 & 0 & -6 & 0 \\
\hline & & $(1,1,1)$ & 0 & 0 & 2 & 0 & 2 & 0 & -2 & $(1,3)$ & 2 & 0 & 2 & 0 \\
\hline & & $(1,1,1)$ & 0 & 0 & -2 & 0 & 2 & 0 & -2 & $(1, \overline{3})$ & -2 & 0 & -2 & 0 \\
\hline $\mathrm{f}$ & $S+$ & $(1,1,1)$ & 0 & 0 & 0 & -2 & -2 & -2 & 0 & $(1,1)$ & -2 & 0 & 6 & 0 \\
\hline
\end{tabular}


Table 5 continued

\begin{tabular}{|c|c|c|c|c|c|c|c|c|c|c|c|c|c|c|}
\hline$F$ & SEC & $(C ; L ; R)$ & $Q_{C}$ & $Q_{\bar{\eta}^{1}}$ & $Q_{\bar{\eta}^{2}}$ & $Q_{\bar{\eta}^{3}}$ & $Q_{\bar{y}^{3,6}}$ & $Q_{\bar{y}^{1} \bar{w}^{5}}$ & $Q_{\bar{w}^{2,4}}$ & $S U(3)_{H_{1,2}}$ & $Q_{\bar{\Phi}^{1}}$ & $Q_{8}$ & $Q_{9}$ & $Q_{\bar{\Phi}^{8}}$ \\
\hline & $b_{1}+b_{2}$ & $(1,1,1)$ & 0 & 0 & 0 & 2 & -2 & -2 & 0 & $(1,1)$ & 2 & 0 & -6 & 0 \\
\hline & $+\alpha+2 \gamma$ & $(1,1,1)$ & 0 & 0 & 0 & 2 & -2 & -2 & 0 & $(1,3)$ & 2 & 0 & 2 & 0 \\
\hline & & $(1,1,1)$ & 0 & 0 & 0 & -2 & -2 & -2 & 0 & $(1, \overline{3})$ & -2 & 0 & -2 & 0 \\
\hline \multirow[t]{4}{*}{$\mathrm{b}$} & $b_{1}+b_{2}$ & $(1,1,1)$ & 0 & 0 & 0 & -2 & 2 & -2 & 0 & $(1,1)$ & -2 & 0 & 6 & 0 \\
\hline & $+\alpha+2 \gamma$ & $(1,1,1)$ & 0 & 0 & 0 & 2 & 2 & -2 & 0 & $(1,1)$ & 2 & 0 & -6 & 0 \\
\hline & & $(1,1,1)$ & 0 & 0 & 0 & 2 & 2 & -2 & 0 & $(1,3)$ & 2 & 0 & 2 & 0 \\
\hline & & $(1,1,1)$ & 0 & 0 & 0 & -2 & 2 & -2 & 0 & $(1, \overline{3})$ & -2 & 0 & -2 & 0 \\
\hline \multirow[t]{4}{*}{$\mathrm{f}$} & $S+$ & $(1,1,1)$ & 0 & -2 & 0 & 0 & 0 & -2 & -2 & $(1,1)$ & -2 & 0 & 6 & 0 \\
\hline & $b_{2}+b_{3}$ & $(1,1,1)$ & 0 & 2 & 0 & 0 & 0 & -2 & -2 & $(1,1)$ & 2 & 0 & -6 & 0 \\
\hline & $+\alpha+2 \gamma$ & $(1,1,1)$ & 0 & 2 & 0 & 0 & 0 & -2 & -2 & $(1,3)$ & 2 & 0 & 2 & 0 \\
\hline & & $(1,1,1)$ & 0 & -2 & 0 & 0 & 0 & -2 & -2 & $(1, \overline{3})$ & -2 & 0 & -2 & 0 \\
\hline \multirow[t]{4}{*}{$\mathrm{b}$} & $b_{2}+b_{3}$ & $(1,1,1)$ & 0 & -2 & 0 & 0 & 0 & 2 & -2 & $(1,1)$ & -2 & 0 & 6 & 0 \\
\hline & $+\alpha+2 \gamma$ & $(1,1,1)$ & 0 & 2 & 0 & 0 & 0 & 2 & -2 & $(1,1)$ & 2 & 0 & -6 & 0 \\
\hline & & $(1,1,1)$ & 0 & 2 & 0 & 0 & 0 & 2 & -2 & $(1,3)$ & 2 & 0 & 2 & 0 \\
\hline & & $(1,1,1)$ & 0 & -2 & 0 & 0 & 0 & 2 & -2 & $(1, \overline{3})$ & -2 & 0 & -2 & 0 \\
\hline \multirow[t]{4}{*}{ f } & $S+$ & $(1,1,1)$ & 0 & 0 & 0 & -2 & -2 & -2 & 0 & $(1,1)$ & 0 & 6 & 0 & 2 \\
\hline & $1+b_{3}$ & $(1,1,1)$ & 0 & 0 & 0 & 2 & -2 & -2 & 0 & $(1,1)$ & 0 & -6 & 0 & -2 \\
\hline & $+\alpha+2 \gamma$ & $(1,1,1)$ & 0 & 0 & 0 & 2 & -2 & -2 & 0 & $(3,1)$ & 0 & 2 & 0 & -2 \\
\hline & & $(1,1,1)$ & 0 & 0 & 0 & -2 & -2 & -2 & 0 & $(\overline{3}, 1)$ & 0 & -2 & 0 & 2 \\
\hline \multirow[t]{4}{*}{$\mathrm{b}$} & $1+b_{3}$ & $(1,1,1)$ & 0 & 0 & 0 & -2 & 2 & -2 & 0 & $(1,1)$ & 0 & 6 & 0 & 2 \\
\hline & $+\alpha+2 \gamma$ & $(1,1,1)$ & 0 & 0 & 0 & 2 & 2 & -2 & 0 & $(1,1)$ & 0 & -6 & 0 & -2 \\
\hline & & $(1,1,1)$ & 0 & 0 & 0 & 2 & 2 & -2 & 0 & $(3,1)$ & 0 & 2 & 0 & -2 \\
\hline & & $(1,1,1)$ & 0 & 0 & 0 & -2 & 2 & -2 & 0 & $(\overline{3}, 1)$ & 0 & -2 & 0 & 2 \\
\hline
\end{tabular}

Table 6 The table displays all the massless sectors for which the "would-be superpartners" are massive and do not form part of the massless spectrum. The "would-be superpartners" arise from the sectors that are obtained by adding the basis vector $S$ to a given sector and are the fermionic counterparts

\begin{tabular}{|c|c|c|c|c|c|c|c|c|c|c|c|c|c|c|}
\hline$F$ & SEC & $(C ; L ; R)$ & $Q_{C}$ & $Q_{\bar{\eta}^{1}}$ & $Q_{\bar{\eta}^{2}}$ & $Q_{\bar{\eta}^{3}}$ & $Q_{\bar{y}^{3,6}}$ & $Q_{\bar{y}^{1} \bar{w}^{5}}$ & $Q_{\bar{w}^{2,4}}$ & $S U(3)_{H_{1,2}}$ & $Q_{\bar{\Phi}^{1}}$ & $Q_{8}$ & $Q_{9}$ & $Q_{\bar{\Phi}^{8}}$ \\
\hline \multirow[t]{8}{*}{$\mathrm{b}$} & \multirow[t]{8}{*}{$\alpha \pm \gamma$} & $(1,1,1)$ & -3 & 1 & 1 & 1 & -2 & 0 & -2 & $(1,1)$ & 2 & -3 & 3 & 0 \\
\hline & & $(1,1,1)$ & -3 & 1 & 1 & 1 & 2 & 0 & 2 & $(1,1)$ & 2 & -3 & 3 & 0 \\
\hline & & $(1,1,1)$ & -3 & 1 & 1 & 1 & 2 & 0 & 2 & $(1,1)$ & 2 & -3 & 3 & 0 \\
\hline & & $(1,1,1)$ & -3 & 1 & 1 & 1 & -2 & 0 & -2 & $(1,1)$ & 2 & -3 & 3 & 0 \\
\hline & & $(1,1,1)$ & 3 & -1 & -1 & -1 & -2 & 0 & -2 & $(1,1)$ & -2 & 3 & -3 & 0 \\
\hline & & $(1,1,1)$ & 3 & -1 & -1 & -1 & 2 & 0 & 2 & $(1,1)$ & -2 & 3 & -3 & 0 \\
\hline & & $(1,1,1)$ & 3 & -1 & -1 & -1 & 2 & 0 & 2 & $(1,1)$ & -2 & 3 & -3 & 0 \\
\hline & & $(1,1,1)$ & 3 & -1 & -1 & -1 & -2 & 0 & -2 & $(1,1)$ & -2 & 3 & -3 & 0 \\
\hline \multirow[t]{8}{*}{$\mathrm{b}$} & \multirow[t]{8}{*}{$\beta \pm \gamma$} & $(1,1,1)$ & -3 & 1 & 1 & 1 & 0 & 2 & 2 & $(1,1)$ & -2 & -3 & 3 & 0 \\
\hline & & $(1,1,1)$ & -3 & 1 & 1 & 1 & 0 & -2 & -2 & $(1,1)$ & -2 & -3 & 3 & 0 \\
\hline & & $(1,1,1)$ & -3 & 1 & 1 & 1 & 0 & -2 & -2 & $(1,1)$ & -2 & -3 & 3 & 0 \\
\hline & & $(1,1,1)$ & -3 & 1 & 1 & 1 & 0 & 2 & 2 & $(1,1)$ & -2 & -3 & 3 & 0 \\
\hline & & $(1,1,1)$ & 3 & -1 & -1 & -1 & 0 & 2 & 2 & $(1,1)$ & 2 & 3 & -3 & 0 \\
\hline & & $(1,1,1)$ & 3 & -1 & -1 & -1 & 0 & -2 & -2 & $(1,1)$ & 2 & 3 & -3 & 0 \\
\hline & & $(1,1,1)$ & 3 & -1 & -1 & -1 & 0 & -2 & -2 & $(1,1)$ & 2 & 3 & -3 & 0 \\
\hline & & $(1,1,1)$ & 3 & -1 & -1 & -1 & 0 & 2 & 2 & $(1,1)$ & 2 & 3 & -3 & 0 \\
\hline
\end{tabular}


Table 6 continued

\begin{tabular}{|c|c|c|c|c|c|c|c|c|c|c|c|c|c|c|}
\hline$F$ & SEC & $(C ; L ; R)$ & $Q_{C}$ & $Q_{\bar{\eta}^{1}}$ & $Q_{\bar{\eta}^{2}}$ & $Q_{\bar{\eta}^{3}}$ & $Q_{\bar{y}^{3,6}}$ & $Q_{\bar{y}^{1} \bar{w}^{5}}$ & $Q_{\bar{w}^{2,4}}$ & $S U(3)_{H_{1,2}}$ & $Q_{\bar{\Phi}^{1}}$ & $Q_{8}$ & $Q_{9}$ & $Q_{\bar{\Phi}^{8}}$ \\
\hline \multirow[t]{8}{*}{$\mathrm{b}$} & $1+b_{1}$ & $(1,1,1)$ & -3 & 1 & 1 & 1 & 0 & 2 & 2 & $(1,1)$ & 0 & 3 & -3 & 2 \\
\hline & $+b_{2}+b_{3}$ & $(1,1,1)$ & -3 & 1 & 1 & 1 & 0 & -2 & -2 & $(1,1)$ & 0 & 3 & -3 & 2 \\
\hline & $+\beta \pm \gamma$ & $(1,1,1)$ & -3 & 1 & 1 & 1 & 0 & -2 & -2 & $(1,1)$ & 0 & 3 & -3 & 2 \\
\hline & & $(1,1,1)$ & -3 & 1 & 1 & 1 & 0 & 2 & 2 & $(1,1)$ & 0 & 3 & -3 & 2 \\
\hline & & $(1,1,1)$ & 3 & -1 & -1 & -1 & 0 & 2 & 2 & $(1,1)$ & 0 & -3 & 3 & -2 \\
\hline & & $(1,1,1)$ & 3 & -1 & -1 & -1 & 0 & -2 & -2 & $(1,1)$ & 0 & -3 & 3 & -2 \\
\hline & & $(1,1,1)$ & 3 & -1 & -1 & -1 & 0 & -2 & -2 & $(1,1)$ & 0 & -3 & 3 & -2 \\
\hline & & $(1,1,1)$ & 3 & -1 & -1 & -1 & 0 & 2 & 2 & $(1,1)$ & 0 & -3 & 3 & -2 \\
\hline \multirow[t]{8}{*}{ b } & $1+b_{1}$ & $(1,1,1)$ & -3 & 1 & 1 & 1 & 2 & 0 & -2 & $(1,1)$ & 0 & 3 & -3 & -2 \\
\hline & $+b_{2}+b_{3}$ & $(1,1,1)$ & -3 & 1 & 1 & 1 & -2 & 0 & 2 & $(1,1)$ & 0 & 3 & -3 & -2 \\
\hline & $+\alpha \pm \gamma$ & $(1,1,1)$ & -3 & 1 & 1 & 1 & 2 & 0 & -2 & $(1,1)$ & 0 & 3 & -3 & -2 \\
\hline & & $(1,1,1)$ & -3 & 1 & 1 & 1 & -2 & 0 & 2 & $(1,1)$ & 0 & 3 & -3 & -2 \\
\hline & & $(1,1,1)$ & 3 & -1 & -1 & -1 & 2 & 0 & -2 & $(1,1)$ & 0 & -3 & 3 & 2 \\
\hline & & $(1,1,1)$ & 3 & -1 & -1 & -1 & -2 & 0 & 2 & $(1,1)$ & 0 & -3 & 3 & 2 \\
\hline & & $(1,1,1)$ & 3 & -1 & -1 & -1 & 2 & 0 & -2 & $(1,1)$ & 0 & -3 & 3 & 2 \\
\hline & & $(1,1,1)$ & 3 & -1 & -1 & -1 & -2 & 0 & 2 & $(1,1)$ & 0 & -3 & 3 & 2 \\
\hline
\end{tabular}

Table 7 Vector-like exotic states. All sectors, fermionic and bosonic, have CPT conjugates which are not displayed

\begin{tabular}{|c|c|c|c|c|c|c|c|c|c|c|c|c|c|c|}
\hline$F$ & SEC & $(C ; L ; R)$ & $Q_{C}$ & $Q_{\bar{\eta}^{1}}$ & $Q_{\bar{\eta}^{2}}$ & $Q_{\bar{\eta}^{3}}$ & $Q_{\bar{y}^{3,6}}$ & $Q_{\bar{y}^{1} \bar{w}^{5}}$ & $Q_{\bar{w}^{2,4}}$ & $S U(3)_{H_{1,2}}$ & $Q_{\bar{\Phi}^{1}}$ & $Q_{8}$ & $Q_{9}$ & $Q_{\bar{\Phi}^{8}}$ \\
\hline \multirow[t]{8}{*}{$f$} & $S+$ & $(1,1,1)$ & -3 & -3 & -1 & -1 & 0 & 0 & 0 & $(1,1)$ & -2 & -3 & 3 & 0 \\
\hline & $b_{2}+b_{3}$ & $(1,1,1)$ & -3 & 1 & 3 & -1 & 0 & 0 & 0 & $(1,1)$ & -2 & -3 & 3 & 0 \\
\hline & $+\beta \pm \gamma$ & $(1,1,1)$ & -3 & 1 & -1 & 3 & 0 & 0 & 0 & $(1,1)$ & -2 & -3 & 3 & 0 \\
\hline & & $(1,1,1)$ & 3 & -1 & 1 & 1 & 0 & 0 & 0 & $(1, \overline{3})$ & -2 & 3 & 1 & 0 \\
\hline & & $(1,1,1)$ & 3 & 3 & 1 & 1 & 0 & 0 & 0 & $(1,1)$ & 2 & 3 & -3 & 0 \\
\hline & & $(1,1,1)$ & 3 & -1 & -3 & 1 & 0 & 0 & 0 & $(1,1)$ & 2 & 3 & -3 & 0 \\
\hline & & $(1,1,1)$ & 3 & -1 & 1 & -3 & 0 & 0 & 0 & $(1,1)$ & 2 & 3 & -3 & 0 \\
\hline & & $(1,1,1)$ & -3 & 1 & -1 & -1 & 0 & 0 & 0 & $(1,3)$ & 2 & -3 & -1 & 0 \\
\hline \multirow[t]{4}{*}{ b } & $b_{2}+b_{3}$ & $(\overline{3}, 1,1)$ & 1 & 1 & -1 & -1 & 0 & 0 & 0 & $(1,1)$ & -2 & -3 & 3 & 0 \\
\hline & $+\beta \pm \gamma$ & $(1,1,1)$ & -3 & 1 & -1 & -1 & 0 & 0 & 0 & $(\overline{3}, 1)$ & 2 & 1 & 3 & 0 \\
\hline & & $(3,1,1)$ & -1 & -1 & 1 & 1 & 0 & 0 & 0 & $(1,1)$ & 2 & 3 & -3 & 0 \\
\hline & & $(1,1,1)$ & 3 & -1 & 1 & 1 & 0 & 0 & 0 & $(3,1)$ & -2 & -1 & -3 & 0 \\
\hline \multirow[t]{8}{*}{$\mathrm{f}$} & $S+$ & $(1,1,1)$ & -3 & 3 & 1 & -1 & 0 & 0 & 0 & $(1,1)$ & 2 & -3 & 3 & 0 \\
\hline & $b_{1}+b_{3}$ & $(1,1,1)$ & -3 & -1 & -3 & -1 & 0 & 0 & 0 & $(1,1)$ & 2 & -3 & 3 & 0 \\
\hline & $+\alpha \pm \gamma$ & $(1,1,1)$ & -3 & -1 & 1 & 3 & 0 & 0 & 0 & $(1,1)$ & 2 & -3 & 3 & 0 \\
\hline & & $(1,1,1)$ & 3 & 1 & -1 & 1 & 0 & 0 & 0 & $(1, \overline{3})$ & 2 & 3 & 1 & 0 \\
\hline & & $(1,1,1)$ & 3 & -3 & -1 & 1 & 0 & 0 & 0 & $(1,1)$ & -2 & 3 & -3 & 0 \\
\hline & & $(1,1,1)$ & 3 & 1 & 3 & 1 & 0 & 0 & 0 & $(1,1)$ & -2 & 3 & -3 & 0 \\
\hline & & $(1,1,1)$ & 3 & 1 & -1 & -3 & 0 & 0 & 0 & $(1,1)$ & -2 & 3 & -3 & 0 \\
\hline & & $(1,1,1)$ & -3 & -1 & 1 & -1 & 0 & 0 & 0 & $(1,3)$ & -2 & -3 & -1 & 0 \\
\hline \multirow[t]{4}{*}{ b } & $b_{1}+b_{3}$ & $(\overline{3}, 1,1)$ & 1 & -1 & 1 & -1 & 0 & 0 & 0 & $(1,1)$ & 2 & -3 & 3 & 0 \\
\hline & $+\alpha \pm \gamma$ & $(1,1,1)$ & -3 & -1 & 1 & -1 & 0 & 0 & 0 & $(\overline{3}, 1)$ & -2 & 1 & 3 & 0 \\
\hline & & $(3,1,1)$ & -1 & 1 & -1 & 1 & 0 & 0 & 0 & $(1,1)$ & -2 & 3 & -3 & 0 \\
\hline & & $(1,1,1)$ & 3 & 1 & -1 & 1 & 0 & 0 & 0 & $(3,1)$ & 2 & -1 & -3 & 0 \\
\hline
\end{tabular}


Table 8 Vector-like exotic states. All sectors, fermionic and bosonic, have CPT conjugates which are not displayed

\begin{tabular}{|c|c|c|c|c|c|c|c|c|c|c|c|c|c|c|}
\hline$F$ & SEC & $(C ; L ; R)$ & $Q_{C}$ & $Q_{\bar{\eta}^{1}}$ & $Q_{\bar{\eta}^{2}}$ & $Q_{\bar{\eta}^{3}}$ & $Q_{\bar{y}^{3,6}}$ & $Q_{\bar{y}^{1} \bar{w}^{5}}$ & $Q_{\bar{w}^{2,4}}$ & $S U(3)_{H_{1,2}}$ & $Q_{\bar{\Phi}^{1}}$ & $Q_{8}$ & $Q_{9}$ & $Q_{\bar{\Phi}^{8}}$ \\
\hline \multirow[t]{4}{*}{$\mathrm{f}$} & $S+$ & $(\overline{3}, 1,1)$ & -3 & -1 & 1 & -1 & 0 & 0 & 0 & $(1,1)$ & 0 & 3 & -3 & -2 \\
\hline & $1+b_{2}$ & $(1,1,1)$ & 3 & -1 & 1 & -1 & 0 & 0 & 0 & $(1, \overline{3})$ & 0 & 3 & 3 & 2 \\
\hline & \multirow[t]{2}{*}{$+\alpha \pm \gamma$} & $(3,1,1)$ & 3 & 1 & -1 & 1 & 0 & 0 & 0 & $(1,1)$ & 0 & -3 & 3 & 2 \\
\hline & & $(1,1,1)$ & -3 & 1 & -1 & 1 & 0 & 0 & 0 & $(1,3)$ & 0 & -3 & -3 & -2 \\
\hline \multirow[t]{8}{*}{$\mathrm{b}$} & $1+b_{2}$ & $(1,1,1)$ & -3 & 3 & 1 & -1 & 0 & 0 & 0 & $(1,1)$ & 0 & 3 & -3 & -2 \\
\hline & \multirow[t]{7}{*}{$+\alpha \pm \gamma$} & $(1,1,1)$ & -3 & -1 & -3 & -1 & 0 & 0 & 0 & $(1,1)$ & 0 & 3 & -3 & -2 \\
\hline & & $(1,1,1)$ & -3 & -1 & 1 & 3 & 0 & 0 & 0 & $(1,1)$ & 0 & 3 & -3 & -2 \\
\hline & & $(1,1,1)$ & -3 & -1 & 1 & -1 & 0 & 0 & 0 & $(\overline{3}, 1)$ & 0 & -3 & -3 & 2 \\
\hline & & $(1,1,1)$ & 3 & -3 & -1 & 1 & 0 & 0 & 0 & $(1,1)$ & 0 & -3 & 3 & 2 \\
\hline & & $(1,1,1)$ & 3 & 1 & 3 & 1 & 0 & 0 & 0 & $(1,1)$ & 0 & -3 & 3 & 2 \\
\hline & & $(1,1,1)$ & 3 & 1 & -1 & -3 & 0 & 0 & 0 & $(1,1)$ & 0 & -3 & 3 & 2 \\
\hline & & $(1,1,1)$ & 3 & 1 & -1 & 1 & 0 & 0 & 0 & $(3,1)$ & 0 & 3 & 3 & -2 \\
\hline \multirow[t]{8}{*}{ f } & $S+$ & $(1,1,1)$ & -3 & -3 & -1 & -1 & 0 & 0 & 0 & $(1,1)$ & 0 & 3 & -3 & 2 \\
\hline & $1+b_{1}$ & $(1,1,1)$ & -3 & 1 & 3 & -1 & 0 & 0 & 0 & $(1,1)$ & 0 & 3 & -3 & 2 \\
\hline & \multirow[t]{6}{*}{$+\beta \pm \gamma$} & $(1,1,1)$ & -3 & 1 & -1 & 3 & 0 & 0 & 0 & $(1,1)$ & 0 & 3 & -3 & 2 \\
\hline & & $(1,1,1)$ & -3 & 1 & -1 & -1 & 0 & 0 & 0 & $(3,1)$ & 0 & -3 & -3 & -2 \\
\hline & & $(1,1,1)$ & 3 & 3 & 1 & 1 & 0 & 0 & 0 & $(1,1)$ & 0 & -3 & 3 & -2 \\
\hline & & $(1,1,1)$ & 3 & -1 & -3 & 1 & 0 & 0 & 0 & $(1,1)$ & 0 & -3 & 3 & -2 \\
\hline & & $(1,1,1)$ & 3 & -1 & 1 & -3 & 0 & 0 & 0 & $(1,1)$ & 0 & -3 & 3 & -2 \\
\hline & & $(1,1,1)$ & 3 & -1 & 1 & 1 & 0 & 0 & 0 & $(\overline{3}, 1)$ & 0 & 3 & 3 & 2 \\
\hline \multirow[t]{4}{*}{$\mathrm{b}$} & $1+b_{1}$ & $(3,1,1)$ & 3 & 1 & -1 & -1 & 0 & 0 & 0 & $(1,1)$ & 0 & 3 & -3 & 2 \\
\hline & \multirow[t]{3}{*}{$+\beta \pm \gamma$} & $(1,1,1)$ & -3 & 1 & -1 & -1 & 0 & 0 & 0 & $(1,3)$ & 0 & 3 & 3 & -2 \\
\hline & & $(\overline{3}, 1,1)$ & -3 & -1 & 1 & 1 & 0 & 0 & 0 & $(1,1)$ & 0 & -3 & 3 & -2 \\
\hline & & $(1,1,1)$ & 3 & -1 & 1 & 1 & 0 & 0 & 0 & $(1, \overline{3})$ & 0 & -3 & -3 & 2 \\
\hline
\end{tabular}

Table 9 Vector-like exotic states. All sectors, fermionic and bosonic, have CPT conjugates which are not displayed

\begin{tabular}{|c|c|c|c|c|c|c|c|c|c|c|c|c|c|c|}
\hline$F$ & SEC & $(C ; L ; R)$ & $Q_{C}$ & $Q_{\bar{\eta}^{1}}$ & $Q_{\bar{\eta}^{2}}$ & $Q_{\bar{\eta}^{3}}$ & $Q_{\bar{y}^{3,6}}$ & $Q_{\bar{y}^{1} \bar{w}^{5}}$ & $Q_{\bar{w}^{2,4}}$ & $S U(3)_{H_{1,2}}$ & $Q_{\bar{\Phi}^{1}}$ & $Q_{8}$ & $Q_{9}$ & $Q_{\bar{\Phi}^{8}}$ \\
\hline \multirow[t]{4}{*}{$\mathrm{f}$} & $1+b_{2}$ & $(1,2,1)$ & 0 & 0 & -2 & -2 & 2 & 0 & 0 & $(1,1)$ & -2 & 0 & 0 & 2 \\
\hline & \multirow[t]{3}{*}{$+b_{3}+2 \gamma$} & $(1,2,1)$ & 0 & 0 & -2 & -2 & -2 & 0 & 0 & $(1,1)$ & 2 & 0 & 0 & -2 \\
\hline & & $(1,1,2)$ & 0 & 0 & 2 & 2 & 2 & 0 & 0 & $(1,1)$ & 2 & 0 & 0 & -2 \\
\hline & & $(1,1,2)$ & 0 & 0 & 2 & 2 & -2 & 0 & 0 & $(1,1)$ & -2 & 0 & 0 & 2 \\
\hline \multirow[t]{4}{*}{$\mathrm{b}$} & $S+$ & $(1,2,1)$ & 0 & 0 & 2 & 2 & -2 & 0 & 0 & $(1,1)$ & -2 & 0 & 0 & 2 \\
\hline & $1+b_{2}$ & $(1,2,1)$ & 0 & 0 & 2 & 2 & 2 & 0 & 0 & $(1,1)$ & 2 & 0 & 0 & -2 \\
\hline & \multirow[t]{2}{*}{$+b_{3}+2 \gamma$} & $(1,1,2)$ & 0 & 0 & -2 & -2 & -2 & 0 & 0 & $(1,1)$ & 2 & 0 & 0 & -2 \\
\hline & & $(1,1,2)$ & 0 & 0 & -2 & -2 & 2 & 0 & 0 & $(1,1)$ & -2 & 0 & 0 & 2 \\
\hline \multirow[t]{4}{*}{$\mathrm{f}$} & $1+b_{1}$ & $(1,2,1)$ & 0 & -2 & 0 & -2 & 0 & 2 & 0 & $(1,1)$ & -2 & 0 & 0 & 2 \\
\hline & \multirow[t]{3}{*}{$+b_{3}+2 \gamma$} & $(1,2,1)$ & 0 & -2 & 0 & -2 & 0 & -2 & 0 & $(1,1)$ & 2 & 0 & 0 & -2 \\
\hline & & $(1,1,2)$ & 0 & 2 & 0 & 2 & 0 & 2 & 0 & $(1,1)$ & 2 & 0 & 0 & -2 \\
\hline & & $(1,1,2)$ & 0 & 2 & 0 & 2 & 0 & -2 & 0 & $(1,1)$ & -2 & 0 & 0 & 2 \\
\hline \multirow[t]{4}{*}{$\mathrm{b}$} & $S+$ & $(1,2,1)$ & 0 & 2 & 0 & 2 & 0 & -2 & 0 & $(1,1)$ & -2 & 0 & 0 & 2 \\
\hline & $1+b_{1}$ & $(1,2,1)$ & 0 & 2 & 0 & 2 & 0 & 2 & 0 & $(1,1)$ & 2 & 0 & 0 & -2 \\
\hline & \multirow[t]{2}{*}{$+b_{3}+2 \gamma$} & $(1,1,2)$ & 0 & -2 & 0 & -2 & 0 & -2 & 0 & $(1,1)$ & 2 & 0 & 0 & -2 \\
\hline & & $(1,1,2)$ & 0 & -2 & 0 & -2 & 0 & 2 & 0 & $(1,1)$ & -2 & 0 & 0 & 2 \\
\hline
\end{tabular}


Table 9 continued

\begin{tabular}{|c|c|c|c|c|c|c|c|c|c|c|c|c|c|c|}
\hline$F$ & SEC & $(C ; L ; R)$ & $Q_{C}$ & $Q_{\bar{\eta}^{1}}$ & $Q_{\bar{\eta}^{2}}$ & $Q_{\bar{\eta}^{3}}$ & $Q_{\bar{y}^{3}, 6}$ & $Q_{\bar{y}^{1} \bar{w}^{5}}$ & $Q_{\bar{w}^{2,4}}$ & $S U(3)_{H_{1,2}}$ & $Q_{\bar{\Phi}^{1}}$ & $Q_{8}$ & $Q_{9}$ & $Q_{\bar{\Phi}^{8}}$ \\
\hline \multirow[t]{4}{*}{$\mathrm{f}$} & $1+b_{1}$ & $(1,2,1)$ & 0 & -2 & -2 & 0 & 0 & 0 & 2 & $(1,1)$ & -2 & 0 & 0 & 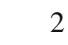 \\
\hline & $+b_{2}+2 \gamma$ & $(1,2,1)$ & 0 & -2 & -2 & 0 & 0 & 0 & -2 & $(1,1)$ & 2 & 0 & 0 & -2 \\
\hline & & $(1,1,2)$ & 0 & 2 & 2 & 0 & 0 & 0 & 2 & $(1,1)$ & 2 & 0 & 0 & -2 \\
\hline & & $(1,1,2)$ & 0 & 2 & 2 & 0 & 0 & 0 & -2 & $(1,1)$ & -2 & 0 & 0 & 2 \\
\hline \multirow[t]{4}{*}{$\mathrm{b}$} & $S+$ & $(1,2,1)$ & 0 & 2 & 2 & 0 & 0 & 0 & -2 & $(1,1)$ & -2 & 0 & 0 & 2 \\
\hline & $1+b_{1}$ & $(1,2,1)$ & 0 & 2 & 2 & 0 & 0 & 0 & 2 & $(1,1)$ & 2 & 0 & 0 & -2 \\
\hline & $+b_{2}+2 \gamma$ & $(1,1,2)$ & 0 & -2 & -2 & 0 & 0 & 0 & -2 & $(1,1)$ & 2 & 0 & 0 & -2 \\
\hline & & $(1,1,2)$ & 0 & -2 & -2 & 0 & 0 & 0 & 2 & $(1,1)$ & -2 & 0 & 0 & 2 \\
\hline \multirow[t]{4}{*}{$\mathrm{f}$} & $S+$ & $(1,1,1)$ & -6 & 0 & 0 & -2 & 0 & 0 & 0 & $(1,1)$ & 2 & 0 & 0 & 2 \\
\hline & $1+b_{3}$ & $(\overline{3}, 1,1)$ & -2 & 0 & 0 & 2 & 0 & 0 & 0 & $(1,1)$ & -2 & 0 & 0 & -2 \\
\hline & $+\alpha+\beta$ & $(1,1,1)$ & 6 & 0 & 0 & 2 & 0 & 0 & 0 & $(1,1)$ & -2 & 0 & 0 & -2 \\
\hline & $+2 \gamma$ & $(3,1,1)$ & 2 & 0 & 0 & -2 & 0 & 0 & 0 & $(1,1)$ & 2 & 0 & 0 & 2 \\
\hline \multirow[t]{4}{*}{$\mathrm{b}$} & $1+b_{3}$ & $(1,1,1)$ & 6 & 0 & 0 & 2 & 0 & 0 & 0 & $(1,1)$ & -2 & 0 & 0 & -2 \\
\hline & $+\alpha+\beta$ & $(1,1,1)$ & -6 & 0 & 0 & -2 & 0 & 0 & 0 & $(1,1)$ & 2 & 0 & 0 & 2 \\
\hline & $+2 \gamma$ & $(3,1,1)$ & 2 & 0 & 0 & -2 & 0 & 0 & 0 & $(1,1)$ & 2 & 0 & 0 & 2 \\
\hline & & $(\overline{3}, 1,1)$ & -2 & 0 & 0 & 2 & 0 & 0 & 0 & $(1,1)$ & -2 & 0 & 0 & -2 \\
\hline
\end{tabular}

\section{References}

1. G. Aad et al., ATLAS Collaboration, Phys. Lett. B 716, 1 (2012)

2. S. Chatrchyan et al., [CMS Collaboration], JHEP 1306, 081 (2013)

3. A.E. Faraggi, D.V. Nanopoulos, K. Yuan, Nucl. Phys. B 335, 347 (1990)

4. G.B. Cleaver, A.E. Faraggi, D.V. Nanopoulos, Phys. Lett. B 455, 135 (1999)

5. G.B. Cleaver, A.E. Faraggi, D.V. Nanopoulos, J.W. Walker, Nucl. Phys. B 593, 471 (2001)

6. V. Braun, Y.H. He, B.A. Ovrut, T. Pantev, Phys. Lett. B 618, 252 (2005)

7. O. Lebedev, H.P. Nilles, S. Raby, S. Ramos-Sanchez, M. Ratz, P.K.S. Vaudrevange, A. Wingerter, Phys. Lett. B 645, 88 (2007)

8. M. Blaszczyk, S. Groot Nibbelink, F. Ruehle, M. Trapletti, P.K.S. Vaudrevange, JHEP 1009, 065 (2010)

9. I. Antoniadis, J. Ellis, J. Hagelin, D.V. Nanopoulos, Phys. Lett. B 231, 65 (1989)

10. A.E. Faraggi, Phys. Lett. B 278, 131 (1992)

11. A.E. Faraggi, Nucl. Phys. B 387, 239 (1992)

12. I. Antoniadis, G.K. Leontaris, J. Rizos, Phys. Lett. B 245, 161 (1990)

13. G.K. Leontaris, J. Rizos, Nucl. Phys. B 554, 3 (1999)

14. K. Christodoulides, A.E. Faraggi, J. Rizos, Phys. Lett. B 702, 81 (2011)

15. G.B. Cleaver, A.E. Faraggi, C. Savage, Phys. Rev. D 63, 066001 (2001)

16. G.B. Cleaver, D.J. Clements, A.E. Faraggi, Phys. Rev. D 65, 106003 (2002)

17. G.B. Cleaver, A.E. Faraggi, S.M.E. Nooij, Nucl. Phys. B 672, 64 (2003)

18. A.E. Faraggi, C. Kounnas, S.E.M. Nooij, J. Rizos. arXiv:hep-th/0311058

19. A.E. Faraggi, C. Kounnas, S.E.M. Nooij, J. Rizos, Nucl. Phys. B 695, 41 (2004)
20. B. Assel, C. Christodoulides, A.E. Faraggi, C. Kounnas, J. Rizos, Phys. Lett. B 683, 306 (2010)

21. B. Assel, C. Christodoulides, A.E. Faraggi, C. Kounnas, J. Rizos, Nucl. Phys. B 844, 365 (2011)

22. L. Bernard et al., Nucl. Phys. B 868, 1 (2013)

23. A.E. Faraggi, J. Rizos, H. Sonmez, Nucl. Phys. B 886, 202 (2014)

24. L. Alvarez-Gaume, P.H. Ginsparg, G.W. Moore, C. Vafa, Phys. Lett. B 171, 155 (1986)

25. H. Itoyama, T.R. Taylor, Phys. Lett. B 186, 129 (1987)

26. A.E. Faraggi, M. Tsulaia, Phys. Lett. B 683, 314 (2010)

27. K.R. Dienes, Phys. Rev. Lett. 65, 1979 (1990)

28. K.R. Dienes, Nucl. Phys. B 429, 533 (1994)

29. K.R. Dienes, A.E. Faraggi, Nucl. Phys. B 457, 409 (1996)

30. J.D. Blum, K.R. Dienes, Nucl. Phys. B 516, 83 (1998)

31. A.E. Faraggi, M. Tsulaia, Eur. Phys. J. C 54, 495 (2008)

32. M. Blaszczyk, S. Groot Nibbelink, O. Loukas, S. Ramos-Sanchez, JHEP 1410, 119 (2014)

33. S. Groot Nobbelink. arXiv: 1502.03604

34. A.E. Faraggi, C. Kounnas, H. Partouche. arXiv:1410.6147

35. S. Abel, K.R. Dienes, E. Mavroudi. arXiv:1502.03087

36. C. Angelantonj, I. Florakis, M. Tsulaia, Phys. Lett. B 736, 365 (2014)

37. I. Florakis. arXiv: 1502.07537

38. A. Lukas, Z. Lalak, E.E. Svanes. arXiv:1504.06978 [hep-th]

39. P.H. Ginsparg, Nucl. Phys. B 295, 153 (1988)

40. J. Bagger, D. Nemechansky, N. Seiberg, S. Yankielowicz, Nucl. Phys. B 289, 53 (1987)

41. D. Chang, A. Kumar, Phys. Rev. D 38, 1893 (1988)

42. D. Chang, A. Kumar, Phys. Rev. D 38, 3734 (1988)

43. I. Antoniadis, C. Bachas, C. Kounnas, Nucl. Phys. B 289, 87 (1987)

44. H. Kawai, D.C. Lewellen, S.H.-H. Tye, Nucl. Phys. B 288, 1 (1987)

45. I. Antoniadis, C. Bachas, Nucl. Phys. B 298, 586 (1988)

46. A.E. Faraggi, C. Kounnas, J. Rizos, Phys. Lett. B 648, 84 (2007)

47. A.E. Faraggi, C. Kounnas, J. Rizos, Nucl. Phys. B 774, 208 (2007)

48. T. Catelin-Julian, A.E. Faraggi, C. Kounnas, J. Rizos, Nucl. Phys. B 812, 103 (2009) 
49. A.E. Faraggi, Nucl. Phys. B 407, 57 (1993)

50. A.E. Faraggi, Eur. Phys. J. C 49, 803 (2007)

51. A.E. Faraggi, D.V. Nanopoulos, Phys. Rev. D 48, 3288 (1993)

52. A. Gregori, C. Kounnas, J. Rizos, Nucl. Phys. B 549, 16 (1999)

53. A.E. Faraggi, Nucl. Phys. B 728, 83 (2005)

54. G. Cleaver, A.E. Faraggi, E. Manno, C. Timirgaziu, Phys. Rev. D 78, 046009 (2008)

55. A.E. Faraggi, C. Kounnas, J. Rizos, Nucl. Phys. B 799, 19 (2008)

56. C. Angelantonj, A.E. Faraggi, M. Tsulaia, JHEP. 1007, 004 (2010)

57. A.E. Faraggi, I. Florakis, T. Mohaupt, M. Tsulaia, Nucl. Phys. B 848, $332(2011)$

58. P. Athanasopoulos, A.E. Faraggi, D. Gepner, Phys. Lett. B 735, 357 (2014)

59. P. Athanasopoulos. arXiv: 1502.04986 [hep-th]

60. A.E. Faraggi, J.C. Pati, Nucl. Phys. B 526, 21 (1998)

61. A.E. Faraggi, Phys. Lett. B 426, 315 (1998)

62. A.E. Faraggi, Phys. Lett. B 326, 62 (1994)

63. A.E. Faraggi, Phys. Lett. B 544, 207 (2002)

64. E. Kiritsis, C. Kounnas, Nucl. Phys. B 503, 117 (1997)

65. P. Berglund et al., Phys. Lett. B 433, 269 (1998)

66. P. Berglund et al., Int. J. Mod. Phys. A 15, 1345 (2000)

67. R. Donagi, A.E. Faraggi, Nucl. Phys. B 694, 187 (2004)

68. A.E. Faraggi, S. Forste, C. Timirgaziu, JHEP 0608, 057 (2005)

69. R. Donagi, K. Wendland, J. Geom. Phys. 59, 942 (2009)

70. Z. Kakushadze, S.H.H. Tye, Phys. Rev. D 54, 7520 (1996)

71. A.E. Faraggi, Int. J. Mod. Phys. A 14, 1663 (1999)

72. A.E. Faraggi, H. Sonmez, Phys. Rev. D 91, 066006 (2015)

73. H. Sonmez, J. Phys. Conf. Ser. 631, 012081 (2015). arXiv: 1503.01193 [hep-th]
74. D. Senechal, Phys. Rev. D 39, 3717 (1989)

75. K.R. Dienes, Phys. Rev. D 73, 106010 (2006)

76. M.R. Douglas. JHEP 0305, 046 (2003)

77. R. Blumenhagen et al., Nucl. Phys. B 713, 83 (2005)

78. F. Denef, M.R. Douglas, JHEP 0405, 072 (2004)

79. T.P.T. Dijkstra, L. Huiszoon, A.N. Schellekens, Nucl. Phys. B 710, $3(2005)$

80. B.S. Acharya, F. Denef, R. Valadro, JHEP 0506, 056 (2005)

81. P. Anastasopoulos, T.P.T. Dijkstra, E. Kiritsis, A.N. Schellekens, Nucl. Phys. B 759, 83 (2006)

82. M.R. Douglas, W. Taylor, JHEP 0701, 031 (2007)

83. K.R. Dienes, M. Lennek, D. Senechal, V. Wasnik, Phys. Rev. D 75, 126005 (2007)

84. O. Lebedev et al., Phys. Lett. B 645, 88 (2007)

85. E. Kiritsis, M. Lennek, A.N. Schellekens, JHEP 0902, 030 (2009)

86. S. Kalara, J.L. Lopez, D.V. Nanopoulos, Nucl. Phys. B 353, 650 (1991)

87. J. Rizos, K. Tamvakis, Phys. Lett. B 262, 227 (1991)

88. A.E. Faraggi, Nucl. Phys. B 487, 55 (1997)

89. M.B. Green, J.H. Schwarz, Phys. Lett. B 149, 117 (1984)

90. M. Dine, N. Seiberg, E. Witten, Nucl. Phys. B 289, 589 (1987)

91. J.J. Atick, L.J. Dixon, A. Sen, Nucl. Phys. B 292, 109 (1987)

92. G.B. Cleaver, A.E. Faraggi, Int. J. Mod. Phys. A 14, 2335 (1999)

93. P. Fayet, J. Iliopoulos, Phys. Lett. B 251, 461 (1974) 